\title{
FRAME MULTIRESOLUTION ANALYSIS IN DISCRETE SETTINGS ON LOCAL FIELDS OF POSITIVE CHARACTERISTIC
}

\author{
OWAIS AHMAD ${ }^{1, *}$, NEYAZ A. SHEIKH ${ }^{1}$, ABDULLAH A.H. AHMADINI ${ }^{2}$, AND MOBIN AHMAD $^{2}$
}

\begin{abstract}
The main aim of this paper is to develop the theory of frame multiresolution analysis (FMRA) in frequency domain on local field of positive characteristic. We first introduce the notion of shift-invariant subspace and the various characteristics of closed shift-invariant subspaces by their fibres in the frequency domain. We define the frame multiresolution analysis in discrete settings on local fields of positive characteristic (LPFC). Furthermore, we established the properties of multiresolution subspaces, which will provide the quantitative criteria for the construction of FMRAs. We also show that the scaling property of an FMRA also holds for the wavelet subspaces and that the Hilbert space $L^{2}(\mathbb{K})$ can be decomposed into the orthogonal sum of these wavelet subspaces.
\end{abstract}

\section{INTRODUCTION}

Duffin and Schaeffer [14] introduced the concept of frame in seperable Hilbert space while dealing with some deep problems in non-harmonic Fourier series. Frames are basis-like systems that span a vector space but allow for linear dependency, which can be used to reduce noise, find sparse representations, or obtain other desirable features unavailable with orthonormal bases.

During the last two decades, there is a substantial body of work that has been concerned with the construction of wavelets on local fields. Even though the structures and metrics of local fields of zero and positive characteristics are similar, their wavelet and MRA (multiresolution analysis) theory are quite different. For example, R. L. Benedetto and J. J. Benedetto [12] developed a wavelet theory for local fields and related groups. They did not develop the multiresolution analysis (MRA) approach, their method is based on the theory of wavelet sets and only allows the construction of wavelet functions whose Fourier transforms are characteristic functions of some sets. Khrennikov, Shelkovich and Skopina [20] constructed a number of scaling functions generating an MRA of $L^{2}\left(\mathbb{Q}_{p}\right)$. But later on in [10], Albeverio, Evdokimov and Skopina proved that all these scaling functions lead to the same Haar MRA and that there exist

\footnotetext{
${ }^{1}$ Department of Mathematics, National Institute of Technology Srinagar, Jammu And KashMIR, 190006, INDIA

${ }^{2}$ Department of Mathematics, College of Science, Jazan University, Jazan -45142, Saudi ARABIA

*CorResponding AUTHOR

E-mail addresses: siawoahmad@gmail.com, neyaznit@yahoo.co.in, aahmadini@jazanu.edu.sa, msyed@jazanu.edu.sa.

Key words and phrases. frame; wavelet frame; multiresolution analysis; local field; Fourier transform.

Received 14/08/2021.
} 
no other orthogonal test scaling functions generating an MRA except those described in [20]. Some wavelet bases for $L^{2}\left(\mathbb{Q}_{p}\right)$ different from the Haar system were constructed in [9, 15] . These wavelet bases were obtained by relaxing the basis condition in the definition of an MRA and form Riesz bases without any dual wavelet systems. For some related works on wavelets and frames on $\mathbb{Q}_{p}$, we refer to [11, 19,21,22]. On the other hand, Lang [23-25] constructed several examples of compactly supported wavelets for the Cantor dyadic group. Farkov [16,17] has constructed many examples of wavelets for the Vilenkin $p$-groups. Jiang et al. [18] pointed out a method for constructing orthogonal wavelets on local field $\mathbb{K}$ with a constant generating sequence and derived necessary and sufficient conditions for a solution of the refinement equation to generate a multiresolution analysis of $L^{2}(\mathbb{K})$. In the series of papers [1-8, 29-32], we have obtained various results related to wavelet and Gabor frames on local fields.

Continuing our study of wavelet analysis on local fields, we in this paper develop the theory of frame multiresolution analysis (FMRA) in frequency domain on local field of positive characteristic. We first introduce the notion of shift-invariant subspace and the various characteristics of closed shift-invariant subspaces by their fibres in the frequency domain are established. We define the frame multitresolution analysis in discrete settings on local fields of positive characteristic (LPFC). Furthermore, we establishe the properties of multiresolution subspaces, which will provide the quantitative criteria for the construction of FMRAs. We also show that the scaling property of an FMRA also holds for the wavelet subspaces and that the Hilbert space $L^{2}(\mathbb{K})$ can be decomposed into the orthogonal sum of these wavelet subspaces.

The rest of the article is structured as follows.In section 2, we recall the preliminaries about local fields and introduce the notion of shift invariant subspaces, FMRA and the various characteristics of closed shift-invariant subspaces by their fibres in the frequency domain are established. In section 3, we define the frame multitresolution analysis in discrete settings on local fields of positive characteristic (LPFC). Furthermore, we establishe the properties of multiresolution subspaces, which will provide the quantitative criteria for the construction of FMRAs. We also show that the scaling property of an FMRA also holds for the wavelet subspaces and that the Hilbert space $L^{2}(\mathbb{K})$ can be decomposed into the orthogonal sum of these wavelet subspaces.

\section{Preliminaries on Local Fields}

Local fields have attracted the attention of several mathematicians, and have found innumerable applications not only in the number theory, but also in the representation theory, division algebras, quadratic forms and algebraic geometry. As a result, local fields are now consolidated as a part of the standard repertoire of contemporary mathematics. Let $\mathbb{K}$ be a field and a topological space. Then $\mathbb{K}$ is called a local field if both $\mathbb{K}^{+}$and $\mathbb{K}^{*}$ are locally compact Abelian groups, where $\mathbb{K}^{+}$and $\mathbb{K}^{*}$ denote the additive and multiplicative groups of $\mathbb{K}$, respectively. If $\mathbb{K}$ is any field and is endowed with the discrete topology, then $K$ is a local field. Further, if $K$ is connected, then $\mathbb{K}$ is either $\mathbb{R}$ or $\mathbb{C}$. If $K$ is not connected, then it is totally disconnected. Hence by a local field, we mean a field $\mathbb{K}$ which is locally compact, non-discrete and totally disconnected. The $p$-adic fields are examples of local fields. In the rest of this paper, we use 
the symbols $\mathbb{N}, \mathbb{N}_{0}$ and $\mathbb{Z}$ to denote the sets of natural, non-negative integers and integers, respectively.

Let $\mathbb{K}$ be a local field. Let $d x$ be the Haar measure on the locally compact Abelian group $\mathbb{K}^{+}$. If $\alpha \in \mathbb{K}$ and $\alpha \neq 0$, then $d(\alpha x)$ is also a Haar measure. Let $d(\alpha x)=|\alpha| d x$. We call $|\alpha|$ the absolute value of $\alpha$. Moreover, the map $x \rightarrow|x|$ has the following properties: (a) $|x|=0$ if and only if $x=0$; (b) $|x y|=|x||y|$ for all $x, y \in \mathbb{K}$; and (c) $|x+y| \leq \max \{|x|,|y|\}$ for all $x, y \in \mathbb{K}$. Property (c) is called the ultrametric inequality. The set $\mathfrak{D}=\{x \in \mathbb{K}:|x| \leq 1\}$ is called the ring of integers in $\mathbb{K}$. Define $\mathfrak{B}=\{x \in \mathbb{K}:|x|<1\}$. The set $\mathfrak{B}$ is called the prime ideal in $\mathbb{K}$. The prime ideal in $K$ is the unique maximal ideal in $\mathfrak{D}$ and hence as result $\mathfrak{B}$ is both principal and prime. Since the local field $\mathbb{K}$ is totally disconnected, so there exist an element of $\mathfrak{B}$ of maximal absolute value. Let $\mathfrak{p}$ be a fixed element of maximum absolute value in $\mathfrak{B}$. Such an element is called a prime element of $K$. Therefore, for such an ideal $\mathfrak{B}$ in $\mathfrak{D}$, we have $\mathfrak{B}=\langle\mathfrak{p}\rangle=\mathfrak{p} \mathfrak{D}$. As it was proved in [11], the set $\mathfrak{D}$ is compact and open. Hence, $\mathfrak{B}$ is compact and open. Therefore, the residue space $\mathfrak{D} / \mathfrak{B}$ is isomorphic to a finite field $G F(q)$, where $q=p^{k}$ for some prime $p$ and $k \in \mathbb{N}$.

Let $\mathfrak{D}^{*}=\mathfrak{D} \backslash \mathfrak{B}=\{x \in \mathbb{K}:|x|=1\}$. Then, it can be proved that $\mathfrak{D}^{*}$ is a group of units in $\mathbb{K}^{*}$ and if $x \neq 0$, then we may write $x=\mathfrak{p}^{k} x^{\prime}, x^{\prime} \in \mathfrak{D}^{*}$. For a proof of this fact we refer to [11]. Moreover, each $\mathfrak{B}^{k}=\mathfrak{p}^{k} \mathfrak{D}=\left\{x \in K:|x|<q^{-k}\right\}$ is a compact subgroup of $K^{+}$and usually known as the fractional ideals of $K^{+}$. Let $\mathcal{U}=\left\{a_{i}\right\}_{i=0}^{q-1}$ be any fixed full set of coset representatives of $\mathfrak{B}$ in $\mathfrak{D}$, then every element $x \in K$ can be expressed uniquely as $x=\sum_{\ell=k}^{\infty} c_{\ell} \mathfrak{p}^{\ell}$ with $c_{\ell} \in \mathcal{U}$. Let $\chi$ be a fixed character on $K^{+}$that is trivial on $\mathfrak{D}$ but is non-trivial on $\mathfrak{B}^{-1}$. Therefore, $\chi$ is constant on cosets of $\mathfrak{D}$ so if $y \in \mathfrak{B}^{k}$, then $\chi_{y}(x)=\chi(y x), x \in K$. Suppose that $\chi_{u}$ is any character on $K^{+}$, then clearly the restriction $\chi_{u} \mid \mathfrak{D}$ is also a character on $\mathfrak{D}$. Therefore, if $\left\{u(n): n \in \mathbb{N}_{0}\right\}$ is a complete list of distinct coset representative of $\mathfrak{D}$ in $K^{+}$, then, as it was proved in [33], the set $\left\{\chi_{u(n)}: n \in \mathbb{N}_{0}\right\}$ of distinct characters on $\mathfrak{D}$ is a complete orthonormal system on $\mathfrak{D}$.

The Fourier transform $\hat{f}$ of a function $f \in L^{1}(K) \cap L^{2}(\mathbb{K})$ is defined by

$$
\hat{f}(\xi)=\int_{\mathbb{K}} f(x) \overline{\chi_{\xi}(x)} d x
$$

It is noted that

$$
\hat{f}(\xi)=\int_{\mathbb{K}} f(x) \overline{\chi_{\xi}(x)} d x=\int_{\mathbb{K}} f(x) \chi(-\xi x) d x
$$

The properties of the Fourier transform on the local field $K$ are quite similar to those of the Fourier analysis on the real line. In particular, if $f \in L^{1}(K) \cap L^{2}(\mathbb{K})$, then $\hat{f} \in L^{2}(\mathbb{K})$ and $\|\hat{f}\|_{2}=\|f\|_{2}$.

We now impose a natural order on the sequence $\{u(n)\}_{n=0}^{\infty}$. We have $\mathfrak{D} / \mathfrak{B} \cong G F(q)$ where $G F(q)$ is a $c$-dimensional vector space over the field $G F(p)$. We choose a set 
$\left\{1=\zeta_{0}, \zeta_{1}, \zeta_{2}, \ldots, \zeta_{c-1}\right\} \subset \mathfrak{D}^{*}$ such that span $\left\{\zeta_{j}\right\}_{j=0}^{c-1} \cong G F(q)$. For $n \in \mathbb{N}_{0}$ satisfying

$$
0 \leq n<q, n=a_{0}+a_{1} p+\cdots+a_{c-1} p^{c-1}, 0 \leq a_{k}<p, \text { and } k=0,1, \ldots, c-1,
$$

we define

$$
u(n)=\left(a_{0}+a_{1} \zeta_{1}+\cdots+a_{c-1} \zeta_{c-1}\right) \mathfrak{p}^{-1}
$$

Also, for $n=b_{0}+b_{1} q+b_{2} q^{2}+\cdots+b_{s} q^{s}, n \in \mathbb{N}_{0}, 0 \leq b_{k}<q, k=0,1,2, \ldots, s$, we set

$$
u(n)=u\left(b_{0}\right)+u\left(b_{1}\right) \mathfrak{p}^{-1}+\cdots+u\left(b_{s}\right) \mathfrak{p}^{-s} .
$$

This defines $u(n)$ for all $n \in \mathbb{N}_{0}$. In general, it is not true that $u(m+n)=u(m)+u(n)$. But, if $r, k \in \mathbb{N}_{0}$ and $0 \leq s<q^{k}$, then $u\left(r q^{k}+s\right)=u(r) \mathfrak{p}^{-k}+u(s)$. Further, it is also easy to verify that $u(n)=0$ if and only if $n=0$ and $\left\{u(\ell)+u(k): k \in \mathbb{N}_{0}\right\}=\left\{u(k): k \in \mathbb{N}_{0}\right\}$ for a fixed $\ell \in \mathbb{N}_{0}$. Hereafter we use the notation $\chi_{n}=\chi_{u(n)}, n \geq 0$.

By $\Omega$ we denote the test function space on $K$, that is, each function $f$ in $\Omega$ is a finite linear combination of functions of the form $\boldsymbol{\Phi}_{k}(x-h), h \in K, k \in \mathbb{Z}$, where $\boldsymbol{\Phi}_{k}$ is the characteristic function of $\mathcal{B}^{k}$. Then, it is clear that $\Omega$ is dense in $L^{p}(K), 1 \leq p<\infty$, and each function in $\Omega$ is of compact support and so is its Fourier transform.

Let the local field $K$ be of characteristic $p>0$ and $\zeta_{0}, \zeta_{1}, \zeta_{2}, \ldots, \zeta_{c-1}$ be as above. We define a character $\chi$ on $K$ as follows:

$$
\chi\left(\zeta_{\mu} \mathfrak{p}^{-j}\right)= \begin{cases}\exp (2 \pi i / p), & \mu=0 \text { and } j=1, \\ 1, & \mu=1, \ldots, c-1 \text { or } j \neq 1 .\end{cases}
$$

Definition 2.1. A sequence $\left\{f_{k}\right\}_{k=1}^{\infty}$ in a separable Hilbert space $\mathbb{H}$ is called a frame if there exists positive constant $A$ and $B$ such that

$$
A\|f\|^{2} \leq \sum_{k=-\infty}^{\infty}\left|\left\langle f, f_{k}\right\rangle\right|^{2} \leq B\|f\|^{2}, \forall f \in \mathbb{H} .
$$

The constants $A$ and $B$ are called frame bounds. If it is possible to choose $A$ and $B$ such that $A=B$, then it is said to be tight frame.

Definition 2.2. A closed shift invariant subspace $\mathcal{S}$ of $L^{2}(\mathbb{K})$ is said to be generated by $\Omega \subset L^{2}(\mathbb{K})$ if $\mathcal{S}=\overline{\operatorname{span}}\left\{\tau_{u(k)} \varphi: k \in \mathbb{N}_{0}, \varphi \in \Omega\right\}$. The length of the closed shift invariant subspace $\mathcal{S}$ is defined as the minimum cardinality of the generating set $\Omega$ and is denoted by len $\mathcal{S}$. If len $\mathcal{S}$ is finite then $\mathcal{S}$ is said to be finitely generated shift invariant(FSI) space. If len $\mathcal{S}=1$, the $\mathcal{S}$ is called principal shift invariant(PSI) space. Further, the spectrum of a shift invariant space is defined to be

$$
\sigma(\mathcal{S})=\{\xi \in \mathfrak{D}: \widehat{\mathcal{S}}(\xi) \neq\{0\}\}
$$

where $\widehat{\mathcal{S}}(\xi)=\left\{\widehat{f}(\xi+u(k)) \in \ell^{2}\left(\mathbb{N}_{0}\right): f \in \mathcal{S}, k \in \mathbb{N}_{0}\right\}$.

Now we state and prove the lemma concerning the sequences of the form

$$
\left\{\widehat{f}(\xi+u(k)): k \in \mathbb{N}_{0}, \xi \in \mathfrak{D}\right\} .
$$


Lemma 2.1. The mapping $T: L^{2}(\mathbb{K}) \rightarrow L^{2}\left(\mathfrak{D}, \ell^{2}\left(\mathbb{N}_{0}\right)\right)$ defined by

$$
T f(\xi)=\left\{\widehat{f}(\xi+u(k)\}_{k \in \mathbb{N}_{0}}\right.
$$

is an isometry.

Proof. For every $f \in L^{2}(\mathbb{K})$ we have

$$
\begin{aligned}
\|T f\|_{L^{2}\left(\mathfrak{D}, \ell^{2}\left(\mathbb{N}_{0}\right)\right)}^{2} & =\int_{\mathfrak{D}}\|T f(\xi)\|_{\ell^{2}\left(\mathbb{N}_{0}\right)}^{2} d \xi \\
& =\int_{\mathfrak{D}} \sum_{k \in \mathbb{N}_{0}}|\widehat{f}(\xi+u(k))|^{2} d \xi \\
& =\int_{\mathfrak{D}+u(k)}|\widehat{f}(\xi)|^{2} d \xi \\
& =\int_{\mathbb{K}}|\widehat{f}(\xi)|^{2} d \xi \\
& =\|\widehat{f}\|^{2} \\
& =\|f\|^{2} .
\end{aligned}
$$

The above calculation asserts that $T$ is an isometry. It also justifies that $T f(\xi) \in \ell^{2}\left(\mathbb{N}_{0}\right)$ for every $\xi \in \mathfrak{D}$. we shall denote the sequences of the form $\left\{\widehat{f}\left(\xi+u(k): k \in \mathbb{N}_{0}\right\}\right.$ by $\widehat{f}_{\| \xi}$.

Definition 2.3. A mapping

$$
\mathcal{J}: \mathfrak{D} \rightarrow\left\{\text { closed subspaces of } \ell^{2}\left(\mathbb{N}_{0}\right)\right\}
$$

is called a range function. The mapping $\mathcal{J}$ is measurable if the function $\xi \rightarrow\langle P(\xi) u, v\rangle_{\ell^{2}\left(\mathbb{N}_{0}\right)}$ is a measurable function for each $u, v \in \ell^{2}\left(\mathbb{N}_{0}\right)$, where $P(\xi)$ is the associated orthogonal projection from $\ell^{2}\left(\mathbb{N}_{0}\right)$ onto $\mathcal{J}(\xi)$.

Lemma 2.2. A closed subspace $\mathcal{S}$ of $L^{2}(\mathbb{K})$ is shift invariant if and only if

$$
\mathcal{S}=\left\{f \in L^{2}(\mathbb{K}): \widehat{f}_{\| \xi} \in \mathcal{J}(\xi) \text { for a.e. } \xi \in \mathfrak{D}\right\},
$$

where $\mathcal{J}$ is measurable range function. There is a one - one correspondence between $\mathcal{S}$ and $\mathcal{J}$ by identifying range functions which are equal almost everywhere. Furthermore, if $\Omega \subset \mathcal{S}$ is a countable set that generates $\mathcal{S}$, then

$$
\mathcal{J}(\xi)=\overline{\operatorname{span}}\left\{\widehat{\varphi}_{\| \xi}: \varphi \in \Omega\right\} \text { for a.e } \xi \in \mathfrak{D} .
$$

Definition 2.4. For a closed shift invariant subspace $\mathcal{S}$ of $L^{2}(\mathbb{K})$ generated by some countable set $\Omega \subset \mathcal{S}$, The fibre of $\mathcal{S}$ at $\xi$ is denoted by $\widehat{\mathcal{S}}_{\| \xi}$ and is defined as

$$
\widehat{\mathcal{S}}_{\| \xi}=\overline{\operatorname{span}}\left\{\widehat{\varphi}_{\| \xi}: \varphi \in \Omega\right\} .
$$

By Lemma 2.2, the above definition is independent of the generating set for almost every $\xi \in \mathfrak{D}$ and hence is well defined upto sets of measure zero. It immediately follows from Lemma 2.2 
that the fibres of a closed shift invariant subspace $\mathcal{S}$ of $L^{2}(\mathbb{K})$ at $\xi \in \mathfrak{D}$ are closed subspaces of $\ell^{2}\left(\mathbb{N}_{0}\right)$ for almost every $\xi \in \mathfrak{D}$.

Lemma 2.3. Let $\mathcal{S}$ be a closed shift invariant subspace generated by some countable set $\Omega=\left\{\varphi_{n}: n \in \mathbb{N}_{0}\right\} \subset \mathcal{S}$. Then the fibres of $\mathcal{S}$ at $\xi \in \mathfrak{D}$ are closed subspaces of $\ell^{2}\left(\mathbb{N}_{0}\right)$ for almost every $\xi \in \mathfrak{D}$.

Proof For every $n \in \mathbb{N}_{0}$, by Lemma 2.1, there exists a set of measure zero $\Upsilon_{n} \subset \mathfrak{D}$, such that $\widehat{\varphi}_{n \| \xi} \in \ell^{2}\left(\mathbb{N}_{0}\right)$ for $\xi \in \mathfrak{D} \backslash \Upsilon_{n}$. Let $\Upsilon=\bigcup_{n \in \mathbb{N}_{0}} \Upsilon_{n}$, therefore for all $\varphi \in \Omega, \widehat{\varphi}_{\| \xi} \in \ell^{2}\left(\mathbb{N}_{0}\right)$ for $\xi \in \mathfrak{D} \backslash \Upsilon$. Consequently, we have $\operatorname{span}\left\{\widehat{\varphi}_{\| \xi}: \varphi \in \Omega\right\} \in \ell^{2}\left(\mathbb{N}_{0}\right)$ for $\xi \in \mathfrak{D} \backslash \Upsilon$. Since $|\Upsilon|=0$ and $\ell^{2}\left(\mathbb{N}_{0}\right)$ is a complete normed linear space. Thus the result follows.

Now we state the definition of frame multiresolution analysis for $L^{2}(\mathbb{K})$ and some known results

Definition 2.5. Let $K$ be a local field of positive characteristic $p>0$ and let $\mathfrak{p}$ be a prime element of $K$. A frame multiresolution analysus of $L^{2}(\mathbb{K})$ is a sequence of closed subspaces $\left\{V_{j}: j \in \mathbb{Z}\right\}$ of $L^{2}(\mathbb{K})$ satisfying the following properties:

(i) $V_{j} \subset V_{j+1}$ for all $j \in \mathbb{Z}$;

(ii) $\bigcup_{j \in \mathbb{Z}} V_{j}$ is dense in $L^{2}(\mathbb{K})$ and $\bigcap_{j \in \mathbb{Z}} V_{j}=\{0\}$;

(iii) $f(\cdot) \in V_{j}$ if and only if $f\left(\mathfrak{p}^{-1} \cdot\right) \in V_{j+1}$ for all $j \in \mathbb{Z}$;

(iv) the function $f$ lying in $V_{0}$ implies that the collection $\left\{f(\cdot-u(k)): k \in \mathbb{N}_{0}\right\}$ lies in $V_{0}$;

(v) the sequence $\left\{\tau_{u(k)} \varphi: k \in \mathbb{N}_{0}\right\}$ is a frame for the subspace $V_{0}$.

The function $\varphi$ is known as the scaling function while the subspaces $V_{j}^{\prime} s$ are known as multiresolution subspaces.

Lemma 2.4. Let $\left\{\tau_{u(k)} \varphi: k \in \mathbb{N}_{0}\right\}$ be a frame for $V_{0}=\overline{\operatorname{span}}\left\{\tau_{u(k)} \varphi: k \in \mathbb{N}_{0}\right\}$ and

$$
V_{j}=\left\{f \in L^{2}(\mathbb{K}): f\left(\mathfrak{p}^{j} \cdot\right) \in V_{0}\right\}, j \in \mathbb{Z}
$$

Then the sequence $\left\{\varphi_{j, k}: k \in \mathbb{N}_{0}\right\}$, where

$$
\varphi_{j, k}=q^{j / 2} \varphi\left(\mathfrak{p}^{-j} x-u(k)\right)
$$

is a frame for $V_{j}$ with the same bounds as those for $V_{0}$.

Lemma 2.5. Let $\left\{\tau_{u(k)} \varphi: k \in \mathbb{N}_{0}\right\}$ be a frame for $V_{0}=\overline{\operatorname{span}}\left\{\tau_{u(k)} \varphi: k \in \mathbb{N}_{0}\right\}$ and for $j \in \mathbb{Z}$, define $V_{j}$ by $(2.5)$. Then for any function $\psi \in V_{1}$, there exists periodic function $G \in L^{2}(\mathfrak{D})$ such that

$$
\widehat{\psi}\left(\mathfrak{p}^{-1} \xi\right)=q^{1 / 2} G(\xi) \widehat{\varphi}(\xi)
$$

Lemma 2.6. Let $\left\{\tau_{u(k)} \varphi: k \in \mathbb{N}_{0}\right\}$ be a frame for $V_{0}=\overline{\operatorname{span}}\left\{\tau_{u(k)} \varphi: k \in \mathbb{N}_{0}\right\}$ and for $j \in \mathbb{Z}$, define $V_{j}$ by (2.5). Assume that $V_{0} \subseteq V_{1}$ and $\Phi=\|\widehat{\varphi}(\xi-u(k))\|_{\ell^{2}\left(\mathbb{N}_{0}\right)}^{2}$. Then there exists periodic function $\mathfrak{H} \in L^{\infty}(\mathfrak{D})$ such that

$$
\widehat{\varphi}(\xi)=q^{1 / 2} \mathfrak{H}(\mathfrak{p} \xi) \widehat{\varphi}(\mathfrak{p} \xi)
$$


Lemma 2.7. Define $V_{0}=\overline{\operatorname{span}}\left\{\tau_{u(k)} \varphi: k \in \mathbb{N}_{0}\right\}$ and for $j \in \mathbb{Z}$, define $V_{j}$ by (2.5). Then for each $j \in \mathbb{Z}$, the orthogonal complement of $V_{j}$ in $V_{j+1}$ is given by

$$
W_{j}=\left\{f \in L^{2}(\mathbb{K}): f\left(\mathfrak{p}^{j} \cdot\right) \in W_{0}\right\}
$$

Proof. For any $h \in V_{j}$ and $g \in W_{j} \subseteq V_{j+1}$, we have $h\left(\mathfrak{p}^{j} \cdot\right) \in V_{0}$ and $g\left(\mathfrak{p}^{j} \cdot\right) \in V_{1}$. Since $h\left(\mathfrak{p}^{j} \cdot\right)$ is arbitrary in $V_{0}$ and

$$
\left\langle g\left(\mathfrak{p}^{j} \cdot\right), h\left(\mathfrak{p}^{j} \cdot\right)\right\rangle=\int_{\mathbb{K}} g\left(\mathfrak{p}^{j} x\right) \overline{g\left(\mathfrak{p}^{j} x\right)} d x=0
$$

we conclude that $g\left(\mathfrak{p}^{j} \cdot\right)$ lies in $W_{0}$.

\section{Frame Multiresolution Analysis on $\ell^{2}(\mathcal{Z})$}

Let $\mathcal{Z}=\left\{u(n): n \in \mathbb{N}_{0}\right\}$, where $\left\{u(n): n \in \mathbb{N}_{0}\right\}$ is a complete list of coset representation of $\mathfrak{D}$ in $K^{+}$. Define $\ell^{2}(\mathcal{Z})$ as

$$
\ell^{2}(\mathcal{Z})=\left\{z: \mathcal{Z} \rightarrow \mathbb{C}: \sum_{n \in \mathbb{N}_{0}}|z(u(n))|^{2}<\infty\right\} .
$$

Clearly $\ell^{2}(\mathcal{Z})$ is a Hilbert space with inner product defined as

$$
\langle z, w\rangle=\sum_{n \in \mathbb{N}_{0}} z(u(n)) \overline{w(u(n))} .
$$

Definition 3.1. The Fourier transform on $\ell^{2}(\mathcal{Z})$ is the map $\wedge: \ell^{2}(\mathcal{Z}) \rightarrow L^{2}(\mathfrak{D})$ defined by

$$
\hat{z}(\xi)=\sum_{n \in \mathbb{N}_{0}} z(u(n)) \chi_{n}(\xi) \text { for } z \in \ell^{2}(\mathcal{Z})
$$

and the inverse Fourier transform on $L^{2}(\mathfrak{D})$ is the map $\vee: L^{2}(\mathfrak{D}) \rightarrow \ell^{2}(\mathcal{Z})$ defined as

$$
f^{\vee}(u(n))=\left\langle f, \chi_{n}\right\rangle=\int_{\mathfrak{D}} f(x) \overline{\chi_{n}(x) d x} \text { for } f \in L^{2}(\mathfrak{D}) .
$$

For $z \in \ell^{2}(\mathcal{Z})$, we have

$$
\begin{aligned}
(\hat{z})^{\vee}(u(n)) & =\left\langle\hat{z}, \chi_{n}\right\rangle \\
& =\left\langle\sum_{m \in \mathbb{N}_{0}} z(u(m)) \chi_{m}, \chi_{n}\right\rangle \\
& =\sum_{m \in \mathbb{N}_{0}} z(u(m))\left\langle\chi_{m}, \chi_{n}\right\rangle \\
& =z(u(n)),
\end{aligned}
$$

since $\left\{\chi_{n}: n \in \mathbb{N}_{0}\right\}$ is an orthonormal basis for $L^{2}(\mathfrak{D})$, i.e., $(\hat{z})^{\vee}(u(n))=z(u(n))$, for $n \in \mathbb{N}_{0}$. It is clear that the formula defining $\hat{z}$ is an integral periodic function because for $m \in \mathbb{N}_{0}$, we 
have

$$
\begin{aligned}
\hat{z}(\xi+u(m)) & =\sum_{n \in \mathbb{N}_{0}} z(u(n)) \chi_{n}(\xi)(u(m)) \\
& =\sum_{n \in \mathbb{N}_{0}} z(u(n)) \chi_{n}(\xi) \\
& =\hat{z}(\xi)
\end{aligned}
$$

For all $z, w \in \ell^{2}(\mathcal{Z})$, we have Parseval's relation:

$$
\langle z, w\rangle=\sum_{n \in \mathbb{N}_{0}} z(u(n)) \overline{w(u(n))}=\int_{\mathfrak{D}} \hat{z}(\xi) \overline{\hat{w} \xi} d \xi=\langle\hat{z}, \hat{w}\rangle,
$$

and the Plancherel's formula :

$$
\|z\|^{2}=\sum_{n \in \mathbb{N}_{0}}|z(u(n))|^{2}=\int_{\mathfrak{D}}|\hat{z}(\xi)|^{2} d \xi=\|\hat{z}\|^{2} .
$$

Let $\varphi$ be the scaling function for a frame multiresolution analysis $\left\{V_{j}: j \in \mathbb{Z}\right\}$, then by Lemma 2.6, there exists a periodic function $\mathfrak{H} \in L^{\infty}(\mathfrak{D})$ such that (2.8) holds. Further by Lemma 2.4, we observe that

$$
\begin{aligned}
V_{j} & =\overline{\operatorname{span}}\left\{q^{j / 2} \varphi\left(\mathfrak{p}^{-j} \cdot-u(k)\right): k \in \mathbb{N}_{0}\right\} \\
& =\overline{\operatorname{span}}\left\{q^{j / 2} \varphi\left(\mathfrak{p}^{-j}(\cdot-u(k))-u(s)\right) ; k \in \mathbb{N}_{0}, 0 \leq s \leq q^{j}-1\right\} \\
& =\left\{\tau_{u(k)} D_{\mathfrak{p}}^{j} \tau_{u(s)} \varphi: k \in \mathbb{N}_{0}, 0 \leq s \leq q^{j}-1\right\}
\end{aligned}
$$

Since

$$
\begin{aligned}
\left(D_{\mathfrak{p}}^{j} \tau_{u(s)} \varphi\right)^{\wedge}(\xi) & =q^{j / 2} \mathcal{F}\left(\varphi\left(\mathfrak{p}^{-j} \cdot-u(s)\right)(\xi)\right. \\
& =q^{j / 2} \mathcal{F}\left(\varphi\left(\mathfrak{p}^{-j} \cdot\right)(\xi) \chi_{s}\left(\mathfrak{p}^{j} \xi\right)\right. \\
& =q^{-j / 2} \chi_{s}\left(\mathfrak{p}^{j} \xi\right) \hat{\varphi}\left(\mathfrak{p}^{j} \xi\right)
\end{aligned}
$$

therefore, by definition 2.3 ,

$$
\widehat{V}_{j \| \xi}:=\operatorname{span}\left\{\left(D_{\mathfrak{p}}^{j} \tau_{u(s)} \varphi\right)_{\| \xi}^{\wedge}: 0 \leq s \leq q^{j}-1\right\}
$$

and hence

$$
\widehat{V}_{j \| \xi}=\operatorname{span}\left\{\nu_{\xi, j, s}: 0 \leq s \leq q^{j}-1\right\} \text { for } \xi \in \mathfrak{D}
$$

where

$$
\nu_{\xi, j, s}=\left\{q^{j / 2} \chi_{k}\left(\mathfrak{p}^{j} u(s)\right) \hat{\varphi}\left(\mathfrak{p}^{j} \xi+\mathfrak{p}^{j} u(k)\right): k \in \mathbb{N}_{0}\right\} .
$$

Here we would like to examine the sequence spaces $\widehat{V}_{j \| \xi}$ which are fibres of the shift invariant subspaces $V_{j}$ and develop a frame multiresolution analysis (FMRA) of $\ell^{2}(\mathcal{Z})$ using these sequence spaces. we are concerned only with $\xi \in \mathfrak{D}$ such that for every $j \in \mathbb{N}_{0}, \widehat{V}_{j \| \xi}$ is a subspace of $\ell^{2}(\mathcal{Z})$. Such a $\xi$ exists almost everywhere in $\mathfrak{D}$, Also by Lemma 2.3, for each $j \in \mathbb{N}_{0}$, there exists a subset $\Gamma_{j} \subset \mathfrak{D}$ of measure zero such that $\widehat{V}_{j \| \eta}$ is a subspace of $\ell^{2}(\mathcal{Z})$ for all $\eta \in \mathfrak{D} \backslash \Gamma_{j}$ and the union $\Gamma:=\cup_{j=0}^{\infty} \Gamma_{j}$ is a measure zero set. 
For $j \in \mathbb{N}_{0}, 0 \leq s \leq q^{j}-1$, define

$\alpha_{\xi, j, s}:=\left\{\begin{array}{l}q^{j / 2} \widehat{\varphi}\left(\mathfrak{p}^{j} \xi+\mathfrak{p}^{j} u(k)\right), \text { if } u(k)=\mathfrak{p}^{-j} u(p)+u(s), p \in \mathbb{N}_{0} \\ 0, \text { otherwise }\end{array}\right.$

Proposition 3.1. For $j \in \mathbb{N}_{0}$, the sequence spaces $\widehat{V}_{j \| \xi}$ are spanned by $\left\{\alpha_{\xi, j, s}: 0 \leq s \leq q^{j}-1\right\}$.

Proof. Note that for $0 \leq s \leq q^{j}-1$, if $u(k)=\mathfrak{p}^{-j} u(n)+u(\ell)$ for some $\ell=0,1, \ldots, q^{j}-1$, then we have $\chi_{s}\left(\mathfrak{p}^{j} u(k)\right) \hat{\varphi}\left(\mathfrak{p}^{j} \xi+\mathfrak{p}^{j} u(k)\right)=\chi_{s}\left(\mathfrak{p}^{j} u(\ell)\right) \hat{\varphi}\left(\mathfrak{p}^{j} \xi+\mathfrak{p}^{j} u(\ell)+u(n)\right)$. Therefore for $0 \leq s \leq q^{j}-1$

$$
\nu_{\xi, j, s}=\sum_{\ell=0}^{q^{j}-1} \chi_{s}\left(\mathfrak{p}^{j} u(\ell)\right) \alpha_{\xi, j, \ell}
$$

and we have the following matrix equation :

$$
\left[\nu_{\xi, j, r}\right]_{s=0}^{q^{j}-1}=\left[\chi_{s}\left(\mathfrak{p}^{j} u(\ell)\right)\right]_{s, \ell=0}^{q^{j}-1}\left[\alpha_{\xi, j, \ell}\right]_{\ell=0}^{q^{j}-1}
$$

Since $\operatorname{det}\left[\chi_{s}\left(\mathfrak{p}^{j} u(\ell)\right)\right]_{s, \ell=0}^{q^{j}-1} \neq 0$, therefore it follows that

$$
\widehat{V}_{j \| \xi}=\operatorname{span}\left\{\alpha_{\xi, j, s}: 0 \leq s \leq q^{j}-1\right\} .
$$

For $j \in \mathbb{N}_{0}, 0 \leq r \leq q^{j}-1$, we shall denote $\widehat{h}_{\xi, j}(r)=\mathfrak{H}\left(\mathfrak{p}^{j} \xi+\mathfrak{p}^{j} u(s)\right)$ and define $\left[h_{\xi, j}(s)\right]_{r=0}^{q^{j}-1}$ to be the inverse Fourier transform of $\left[\hat{h}_{\xi, j}(r)\right]_{r=0}^{q^{j}-1}$.

Proposition 3.2. For $j \in \mathbb{N}_{0}, 0 \leq s \leq q^{j}-1$, the following scaling equation holds,

$$
\alpha_{\xi, j, s}=\sum_{\ell=0}^{q^{j}-1} \hat{h}_{\xi, j+1}\left(\mathfrak{p}^{-j} u(\ell)+u(s)\right) \alpha_{\xi, j+1, \mathfrak{p}^{-j} u(\ell)+u(s)} .
$$

Proof. For a fixed $j \in \mathbb{N}_{0}$ and a fixed $s=0,1, \ldots, q^{j}-1$, we observe that if $u(k)=\mathfrak{p}^{j} u(n)+$ $u(s)$ for some $n \in \mathbb{N}_{0}$, then

$$
\begin{aligned}
\alpha_{\xi, j, s}(k) & =q^{j / 2} \widehat{\varphi}\left(\mathfrak{p}^{j} \xi+\mathfrak{p}^{j} u(k)\right) \\
& =q^{(j+1) / 2} \mathfrak{H}\left(\mathfrak{p}^{j+1} \xi+\mathfrak{p}^{j+1} u(k)\right) \widehat{\varphi}\left(\mathfrak{p}^{j+1} \xi+\mathfrak{p}^{j+1} u(k)\right) \\
& =\widehat{h}_{\xi, j+1}\left(\mathfrak{p}^{-j} u(t)+u(s)\right) \alpha_{\xi, j+1, \mathfrak{p}^{-j} u(t)+u(s)}(u(k)) \\
& =\sum_{\ell=0}^{q^{j}-1} \widehat{h}_{\xi, j+1}\left(\mathfrak{p}^{-j} u(\ell)+u(s)\right) \alpha_{\xi, j+1, \mathfrak{p}^{-j} u(\ell)+u(s)}(u(k)),
\end{aligned}
$$

since we also have $u(k)=\mathfrak{p}^{-(j+1)} u(r)+\mathfrak{p}^{-j} u(t)+u(s)$ for some $r \in \mathbb{N}_{0}$ and $t=0,1, \ldots, q-1$, where $n=q s+t$ and this representation of $n$ is unique by the division algorithm. For $u(k) \neq$ $\mathfrak{p}^{-j} u(n)+u(s)$, where $n \in \mathbb{N}_{0}$, then both sides of (3.5) are equal to zero at the $k^{t h}$-ordinate.

Let us suppose that there are functions $\psi_{m} \in V_{1}$ for $m=1,2, \ldots, L$, such that $\left\{\tau_{k} \psi_{m}\right.$ : $\left.k \in \mathbb{N}_{0}, m=1,2, \ldots, L\right\}$ spans $W_{0}$, the orthogonal complement of $V_{0}$ in $V_{1}$. Then by Lemma 
2.5 , there exists periodic functions $G_{1}, G_{2}, \ldots, G_{L} \in L^{2}(\mathfrak{D})$ such that

$$
\widehat{\psi}_{m}\left(\mathfrak{p}^{-1} \xi\right)=q^{1 / 2} G_{m}(\xi) \hat{\varphi}(\xi) \text { in } L^{2}(\mathbb{K}) \text { for } 0 \leq m \leq L
$$

For $j \in \mathbb{N}_{0}$, by definition 2.4 and Lemma 2.7,

$$
\begin{aligned}
\widehat{W}_{j \| \xi} & =\operatorname{span}\left\{\left(D_{\mathfrak{p}}^{j} \tau_{u(s)} \psi_{m}\right)_{\| \xi}^{\wedge}: 0 \leq s \leq q^{j}-1, m=1,2, \ldots, L\right\} \\
& =\operatorname{span}\left\{u_{\xi, j, s}^{m}: 0 \leq s \leq q^{j}-1, m=1,2, \ldots, L\right\}, \text { for } \xi \in \mathfrak{D}
\end{aligned}
$$

where

$$
u_{\xi, j, s}^{m}=\left\{q^{j / 2} \chi_{s}\left(\mathfrak{p}^{j} u(k)\right) \widehat{\psi}_{m}\left(\mathfrak{p}^{j} \xi+\mathfrak{p}^{j} u(k)\right): k \in \mathbb{N}_{0}\right\} .
$$

Likewise, we shall restrict $\xi \in \mathfrak{D} \backslash \Gamma$ such that for every $j \in \mathbb{N}_{0}, \widehat{W}_{j \| \xi}$ is a subsequences of $\widehat{V}_{j \| \xi}$. Such a $\xi$ exists almost everywhere in $\mathfrak{D}$. Therefore, there exists a subset $\Lambda_{j} \subset \mathfrak{D} \backslash \Gamma$ of measure zero such that this holds for a given $j \in \mathbb{N}_{0}$ and all $\xi \in \mathfrak{D} \backslash\left(\Gamma \cup \Lambda_{j}\right)$ and the union $\Lambda:=\bigcup_{j=0}^{\infty} \Lambda_{j}$ is a set of measure zero. For $j \in \mathbb{N}_{0}, m=1,2, \ldots, L$ and $0 \leq s \leq q^{j}-1$, define

$\beta_{\xi, j, s}^{m}(u(k)):=\left\{\begin{array}{l}q^{j / 2} \widehat{\psi}_{m}\left(\mathfrak{p}^{j} \xi+\mathfrak{p}^{j} u(k)\right), \text { if } u(k)=\mathfrak{p}^{-j} u(p)+u(s), p \in \mathbb{N}_{0} \\ 0, \text { otherwise. }\end{array}\right.$

Proposition 3.3. For $j \in \mathbb{N}_{0}$, the sequence spaces $\widehat{W}_{j \| \xi}$ are spanned by

$$
\left\{\beta_{\xi, j, s}^{m}: 0 \leq r \leq q^{j}-1,1 \leq m \leq L\right\} .
$$

Proof. The proof is similar to that of Proposition 3.1.

For $j \in \mathbb{N}_{0}, 1 \leq m \leq L$ and $0 \leq r \leq q^{j}-1$, we denote

$$
\widehat{g}_{\xi, j}^{m}(u(r))=G_{m}\left(\mathfrak{p}^{j} \xi+\mathfrak{p}^{j} u(r)\right)
$$

and define $\left[g_{\xi, j}^{m}(u(s))\right]_{s=0}^{q^{j}-1}$ to be the inverse Fourier transform of $\left[\widehat{g}_{\xi, j}^{m}(u(r))\right]_{r=0}^{q^{j}-1}$.

Proposition 3.4. For $j \in \mathbb{N}_{0}$ and $0 \leq s \leq q^{j}-1$, the following equation holds:

$$
\beta_{\xi, j, s}^{m}=\sum_{\ell=0}^{q^{j}-1} \widehat{g}_{\xi, j+1}^{m}\left(\mathfrak{p}^{-j} u(\ell)+u(s)\right) \alpha_{\xi, j+1, \mathfrak{p}^{-j} u(\ell)+u(s)},
$$

for $m=1,2, \ldots, L$.

Proof. The proof is similar to that of Proposition 3.2.

Now we introduce the notion of frame multiresolution analysis (FMRA) on $\ell^{2}(\mathcal{Z})$ and we use it to construct wavelet frames for $\ell^{2}(\mathcal{Z})$.

We shall define the modulation operator and the motivation for defining the operator comes from the fact that shifts in the time domain corresponds to modulations in the frequency domain.

Definition 3.2. For $j \in \mathbb{N}_{0}$, define the modulation operator $\mathcal{E}: \ell^{2}(\mathcal{Z}) \rightarrow \ell^{2}(\mathcal{Z})$ by

$$
\left(\mathcal{E}_{j} \alpha\right)(u(k)):=\chi\left(\mathfrak{p}^{j} u(k)\right) \alpha(u(k)) \text { for } k \in \mathbb{N}_{0} .
$$


we shall proceed with the definition of a frame multiresolution analysis of $\ell^{2}(\mathcal{Z})$ and use it to construct wavelet frames for $\ell^{2}(\mathcal{Z})$.

Definition 3.3. A frame multiresolution analysis of $\ell^{2}(\mathcal{Z})$ consists of a sequence of subspaces $\left\{\widehat{V}_{j}: j \in \mathbb{N}_{0}\right\}$ of $\ell^{2}(\mathcal{Z})$ such that it satisfies the following conditions:

(i) For $j \in \mathbb{N}_{0}, \widehat{V}_{j} \subseteq \hat{V}_{j+1}$.

(ii) $\overline{\bigcup_{j=0}^{\infty} \widehat{V}_{j}}=\ell^{2}(\mathcal{Z})$.

(iii)There exists positive constants $A$ and $B$ such that for every $j \in \mathbb{N}_{0}$, there is a sequence $\widehat{\varphi}_{j} \in \widehat{V}_{j}$ such that the collection $\left\{\mathcal{E}_{j}^{s} \widehat{\varphi}_{j}: 0 \leq s \leq q^{j}-1\right\}$ is a frame for $\widehat{V}_{j}$ with frame bounds $A$ and $B$.

For $j \in \mathbb{N}_{0}$, let $\widehat{\varphi}_{j} \in \ell^{2}(\mathcal{Z})$ and define

$$
\widehat{V}_{j}=\operatorname{span}\left\{\mathcal{E}_{j}^{s} \widehat{\varphi}_{j}: 0 \leq s \leq q^{j}-1\right\} .
$$

and we define the orthogonal sequences $\alpha_{j, s}, 0 \leq s \leq q^{j}-1$, by

$\alpha_{j, s}(u(k)):=\left\{\begin{array}{l}\hat{\varphi}_{j}(u(k)), \text { if } u(k)=\mathfrak{p}^{-j} u(p)+u(s), p \in \mathbb{N}_{0} \\ 0, \text { otherwise }\end{array}\right.$

therefore, we have the decomposition $\widehat{\varphi}_{j}:=\sum_{s=0}^{q^{j}-1} \alpha_{j, s}$.

Proposition 3.5. For $j \in \mathbb{N}_{0}$, the sequence spaces $\widehat{V}_{j}$ are spanned $\left\{\alpha_{j, s}: 0 \leq s \leq q^{j}-1\right\}$.

Proof. For $s=0,1, \ldots, q^{j}-1$, if $u(k)=\mathfrak{p}^{-j} u(n)+u(\ell)$ for some $\ell \in\left\{0,1, \ldots, q^{j}-1\right\}$, then we have

$$
\left(\mathcal{E}_{j}^{s} \hat{\varphi}_{j}\right)(u(k))=\chi_{s}\left(\mathfrak{p}^{j} u(n)+u(\ell)\right) .
$$

Thus for $s=0,1, \ldots, q^{j}-1, \mathcal{E}_{j}^{s} \widehat{\varphi}_{j}=\sum_{\ell=0}^{q^{j}-1} \chi_{s}\left(\mathfrak{p}^{j} u(\ell)\right) \alpha_{j, \ell}$. Consequently,

$$
\left[\mathcal{E}_{j}^{s} \hat{\varphi}_{j}\right]_{s=0}^{q^{j}-1}=\left[\chi_{s}\left(\mathfrak{p}^{j} u(\ell)\right]_{s=0}^{q^{j}-1}\left[\alpha_{j, \ell}\right]_{\ell=0}^{q^{j}-1}\right.
$$

which implies that $\widehat{V}_{j}=\operatorname{span}\left\{\alpha_{j, s}: s=0,1, \ldots, q^{j}-1\right\}$.

Now we would like to characterize when modulations of a set of sequences $\widehat{\vartheta}_{j}^{m}$ for $m=$ $1,2, \ldots, L$, where $j \in \mathbb{N}_{0}$, form a frame for their linear span with the help of the following theorem developed for $L^{2}(\mathfrak{D})$ functions. For $j \in \mathbb{N}_{0}$, we define the $\mathfrak{p}^{j}$ shift operator $\mathcal{T}_{j}$ : $L^{2}(\mathfrak{D}) \rightarrow L^{2}(\mathfrak{D})$ by

$$
\mathcal{T}_{j} f:=f\left(\cdot-\mathfrak{p}^{j}\right)
$$

Theorem 3.1. For $j \in \mathbb{N}_{0}$ and $\vartheta_{j}^{m} \in L^{2}(\mathfrak{D})$ for $m=1,2, \ldots, L$, the collection

$$
\left\{\mathcal{T}_{j}^{s} \vartheta_{j}^{m}: 0 \leq s \leq q^{j}-1,1 \leq m \leq L\right\}
$$


is a frame for its linear span $V$ with frame bounds $A$ and $B$ if and only if for each $s=$ $0,1, \ldots, q^{j}-1$

$$
\mathfrak{p}^{j} A \leq \lambda_{s}^{m} \leq \mathfrak{p}^{j} B
$$

for all nonzero eigenvalues $\lambda_{s}^{m}, m=1,2, \ldots, L$ of $\mathcal{R}_{j}(u(s))=\left[\left\langle f_{j, s}^{m}, f_{j, s}^{n}\right\rangle_{L^{2}(\mathfrak{D})}\right]_{m, n=1}^{L}$,

where $f_{j, s}^{m}=\sum_{p \in \mathbb{N}_{0}} \widehat{\vartheta}_{j}^{m}\left[\mathfrak{p}^{j} u(p)+u(r)\right] \overline{\chi\left(\mathfrak{p}^{j} u(p)+u(s)\right)}$ for $m=1,2, \ldots, L$.

Corollary 3.1. For $j \in \mathbb{N}_{0}$ and $\vartheta_{j}^{m} \in L^{2}(\mathcal{Z})$ for $m=1,2, \ldots, L$, the collection

$$
\left\{\mathcal{E}_{j}^{s} \vartheta_{j}^{m}: s=0,1, \ldots, q^{j}-1, m=1,2, \ldots L\right\}
$$

is a frame for its linear span $\widehat{V}$ with frame bounds $A$ and $B$ if and only if for each $s=$ $0,1, \ldots, q^{j}-1$

$$
\mathfrak{p}^{j} A \leq \lambda_{s}^{m} \leq \mathfrak{p}^{j} B
$$

for all nonzero eigenvalues $\lambda_{s}^{m}, m=1,2, \ldots, L$ of $\mathcal{R}_{j}(u(s))=\left[\left\langle\alpha_{j, s}^{m}, \alpha_{j, s}^{n}\right\rangle_{L^{2}(\mathcal{Z})}\right]_{m, n=1}^{L}$,

where $\alpha_{j, s}^{m}$ is defined in a similar manner as in $(3.11)$ for $m=1,2, \ldots, L$.

Proof. By the definition of frame, $\left\{\mathcal{E}_{j}^{s} \vartheta_{j}^{m}: s=0,1, \ldots, q^{j}-1, m=1,2, \ldots L\right\}$ is a frame for $\widehat{V}$ with frame bounds $A$ and $B$ if for every $\widehat{\alpha} \in \widehat{V}$,

$$
A\|\widehat{\alpha}\|_{\ell^{2}(\mathcal{Z})}^{2} \leq \sum_{m=1}^{L} \sum_{s=0}^{q^{j}-1}\left|\left\langle\widehat{\alpha}, \mathcal{E}_{j}^{s} \widehat{\vartheta}_{j}^{m}\right\rangle_{\ell^{2}(\mathcal{Z})}\right|^{2} \leq B\|\widehat{\alpha}\|_{\ell^{2}(\mathcal{Z})}^{2} .
$$

Define the functions $\widehat{\vartheta}_{j}^{m} \in L^{2}(\mathfrak{D})$ for $m=1,2, \ldots, L$ by $\widehat{\vartheta}_{j}^{m}=\sum_{n \in \mathbb{N}_{0}} \widehat{\vartheta}_{j}^{m}(u(n)) \overline{\chi(u(n))}$ and the function $\alpha:=\sum_{n \in \mathbb{N}_{0}} \hat{\alpha}(u(n)) \chi(u(n))$. we also define the functions $f_{j, s}^{m}$ for $m=1,2, \ldots, L$ as in Theorem 4.1. Then (3.15) is equivalent to

$$
A\|\alpha\|_{L^{2}(\mathfrak{D})}^{2} \leq \sum_{m=1}^{L} \sum_{s=0}^{q^{j}-1}\left|\left\langle\alpha, \mathcal{T}_{j}^{s} \vartheta_{j}^{m}\right\rangle_{L^{2}(\mathfrak{D})}\right|^{2} \leq B\|\alpha\|_{L^{2}(\mathfrak{D})}^{2}
$$

that is,

$$
\left\{\mathcal{T}_{j}^{s} \vartheta_{j}^{m}: s=0,1, \ldots, q^{j}-1, m=1,2, \ldots L\right\}
$$

is a frame for its linear span $V$ with frame bounds $A$ and $B$. For $1 \leq m, n \leq L$,

$$
\left\langle f_{j, s}^{m}, f_{j, s}^{n}\right\rangle_{L^{2}(\mathfrak{D})}=\left\langle\alpha_{j, s}^{m}, \alpha_{j, s}^{n}\right\rangle_{\ell^{2}(\mathcal{Z})} .
$$

Therefore, the result follows from Theorem 3.1.

Let us now return to the context of $\widehat{V}_{j}$ of the form (3.10).

Corollary 3.2. For $j \in \mathbb{N}_{0}$, the collection

$$
\left\{\mathcal{E}_{j}^{s} \widehat{\varphi}_{j}: r=0,1, \ldots, q^{j}-1,\right\}
$$

is a frame for $\widehat{V}_{j}$ with frame bounds $A$ and $B$ if and only if for $s \in\left\{0,1, \ldots, q^{j}-1\right\} \backslash\left\{s: \alpha_{j, s}=0\right\}$,

$$
\mathfrak{p}^{j} A \leq\left\|\alpha_{j, s}\right\|_{\ell^{2}\left(\mathbb{N}_{0}\right)}^{2} \leq \mathfrak{p}^{j} B,
$$


In addition, the collection

$$
\left\{\mathfrak{p}^{-j / 2} \alpha_{j, s}: s \in\left\{0,1, \ldots, q^{j}-1\right\} \backslash\left\{s: \alpha_{j, s}=0\right\}\right\}
$$

is an orthogonal basis for $\widehat{V}_{j}$ with frame bounds $A$ and $B$.

Corollary 3.3. For $j \in \mathbb{N}_{0}$

$$
\operatorname{dim} \hat{V}_{j}=q^{j}-\left|N_{j}\right|
$$

where $N_{j}=\left\{r \in\left\{0,1, \ldots, q^{j}-1\right\}: \alpha_{j, r}=0\right\}$.

Proposition 3.6. For $j \in \mathbb{N}_{0}$, the following are equivalent:

(i) $\widehat{V}_{j} \subseteq \hat{V}_{j+1}$.

(ii) There exists $h_{j+1} \in \mathcal{S}\left(\mathfrak{p}^{-(j+1)}\right)$ such that

$$
\widehat{\varphi}_{j}=\sum_{\ell=0}^{q^{j+1}} h_{j+1}(\ell) \mathcal{E}_{j+1}^{\ell} \widehat{\varphi}_{j+1} .
$$

(iii) There exists $\widehat{h}_{j+1} \in \mathcal{S}\left(\mathfrak{p}^{-(j+1)}\right)$ such that for $s=0,1, \ldots, q^{j}$,

$$
\alpha_{j, s}=\sum_{\ell=o}^{q-1} \widehat{h}_{j+1}\left(\mathfrak{p}^{-j} u(\ell)+u(s)\right) \alpha_{j+1, \mathfrak{p}^{-j} \ell+s} .
$$

Proof. (i) $\Rightarrow$ (ii) :The proof of this part follows from the definition of $\widehat{V}_{j}$ 's

(ii) $\Rightarrow$ (iii) For a fixed $j \in \mathbb{N}_{0}$ and a fixed $s=0,1, \ldots, q^{j}-1$, we observe that if $u(k)=$ $\mathfrak{p}^{-j} u(p)+u(r)$ for some $p=q s+t$ where $s \in \mathbb{N}_{0}$ and $t=0,1, \ldots, q-1$, then

$$
\begin{aligned}
\alpha_{j, s}(u(k)) & =\sum_{\ell=0}^{q^{j+1}} h_{j+1}(\ell) \chi_{\ell}\left(\mathfrak{p}^{j+1} u(k)\right) \hat{\varphi}_{j+1}(u(k)) \\
& =\sum_{\ell=0}^{q^{j+1}} h_{j+1}(\ell) \chi_{\ell}\left(\mathfrak{p}^{j+1} u(k)\right) \hat{\varphi}_{j+1, \mathfrak{p}^{-j} \ell+s}(u(k)) \\
& =\widehat{h}_{j+1}\left(\mathfrak{p}^{-j} t+s\right) \alpha_{j+1, \mathfrak{p}-j t+s}(u(k)) \\
& =\sum_{\ell=0}^{q-1} \widehat{h}_{j+1}\left(\mathfrak{p}^{-j} \ell+s\right) \alpha_{j+1, \mathfrak{p}^{-j} \ell+s}(u(k))
\end{aligned}
$$

where $\widehat{h}_{j+1}$ is the Fourier transform of $h_{j+1}$. For $u(k) \neq \mathfrak{p}^{-j} u(p)+u(s)$, where $p \in \mathbb{N}_{0}$, both sides of (3.19) are equal to zero at the $k^{\text {th }}$ ordinate.

(iii) $\Rightarrow$ (i): The proof of this part follows from Proposition 3.5.

Proposition 3.7 For $j \in \mathbb{N}_{0}$, assume that $\widehat{V}_{j} \subseteq \widehat{V}_{j+1}$. Then the sequence spaces $\widehat{V}_{j}, j \in \mathbb{N}_{0}$ are invariant under modulation operator $\mathcal{E}_{k}$ for $k \leq j$. 
Proof. Fix $j \in \mathbb{N}_{0}$ and for any sequence $\alpha \in \widehat{V}_{j}$, there exists $\left[\alpha_{j}(u(s))\right]_{s=0}^{q^{j}-1}$ such that

$$
\alpha=\sum_{s=0}^{q^{j}-1} \alpha_{j}(u(s)) \alpha_{j, s} .
$$

Then for $k \leq j$ and $n \in \mathbb{N}_{0}$,

$$
\begin{aligned}
\left(\mathcal{E}_{k} \alpha\right)(u(n)) & =\sum_{s=0}^{q^{j}-1} \alpha_{j}(u(r)) \chi\left(\mathfrak{p}^{k} u(n)\right) \alpha_{j, s}(u(n)) \\
& =\sum_{s=0}^{q^{j}-1} \alpha_{j}(u(s)) \chi\left(\mathfrak{p}^{k} u(r)\right) \alpha_{j, s}(u(n)) .
\end{aligned}
$$

Therefore $\mathcal{E}_{k} \alpha$ lies in $\widehat{V}_{j}$.

We shall find a sufficient and necessary condition for the density condition of an FMRA to be satisfied.

Lemma 3.1. Suppose that $\alpha \in \ell^{1}(\mathcal{Z})$ and $\sum_{n \in \mathbb{N}_{0}} \alpha\left(\mathfrak{p}^{-j} u(n)+u(r)\right)=0$ for all $j \geq 0$ and $r=$ $0,1, \ldots, q^{j}-1$. Then $a=0$.

Proof. For any $\epsilon>0$, there exists some natural number $N$ such that $\sum_{|s|>N}|\alpha(u(s))|<\epsilon$. For each $s \in \mathbb{Z}$, choose $j \geq 0$ such that $\mathfrak{p}^{-j}>N+|s|$. Consequently,

$$
\begin{aligned}
|\alpha(u(s))| & =\left|\sum_{n \in \mathbb{N}_{0}} \alpha\left(\mathfrak{p}^{-j} u(n)+u(s)\right)-\sum_{n \in \mathbb{N}} \alpha\left(\mathfrak{p}^{-j} u(n)+u(s)\right)\right| \\
& =\mid \sum_{n \in \mathbb{N}} \alpha\left(\mathfrak{p}^{-j} u(n)+u(s) \mid\right. \\
& \leq \sum_{n \in \mathbb{N}}\left|\alpha\left(\mathfrak{p}^{-j} u(n)+u(s)\right)\right|<\epsilon .
\end{aligned}
$$

Lemma 3.2. Let $\widehat{V}$ be a subspace of $\ell^{2}(\mathcal{Z})$ that is invariant under modulation operator $\mathcal{E}_{j}$ for all $j \in \ell^{2}(\mathcal{Z})$, and suppose that $b \in \ell^{2}(\mathcal{Z})$. Then

$$
\langle\alpha, \beta\rangle_{\ell^{2}(\mathcal{Z})}=0 \text { for all } \alpha \in \hat{V}
$$

if and only if

$$
\alpha(u(n)) \overline{\beta(u(n))}=0 \text { for all } n \in \mathbb{N}_{0} \text { and } \alpha \in \hat{V} \text {. }
$$

Proof. $\quad(\Leftarrow)$ This is obvious. 
$(\Rightarrow)$ For any $\alpha \in \widehat{V}, j \in \mathbb{N}_{0}$ and $\ell=0,1, \ldots, q^{j}-1$,

$$
\begin{aligned}
0 & =\left\langle\left\{\chi_{n}\left(\mathfrak{p}^{j} u(\ell)\right) \alpha(u(n))\right\}_{n}, \beta(u(n))\right\rangle_{\ell^{2}(\mathcal{Z})} \\
& =\sum_{n \in \mathbb{N}_{0}} \alpha(u(n)) \overline{\beta u(n)} \chi_{n}\left(\mathfrak{p}^{j} u(\ell)\right) \\
& =\sum_{s=1}^{q^{j}-1} \sum_{n \in \mathbb{N}_{0}} \alpha\left(\mathfrak{p}^{-j} u(n)+u(s) \overline{\beta\left(\mathfrak{p}^{-j} u(n)+u(s)\right)} \chi_{s}\left(\mathfrak{p}^{j} u(\ell)\right)\right. \\
& =\widehat{\gamma}(u(\ell)) .
\end{aligned}
$$

By taking the inverse Fourier transform of (3.21) leads to

$$
\gamma(u(s))=\sum_{n \in \mathbb{N}_{0}} \alpha\left(\mathfrak{p}^{-j} u(n)+u(s)\right) \overline{\beta\left(\mathfrak{p}^{-j} u(n)+u(s)\right)}=0, \text { for all } j \in \mathbb{N}_{0}, s \in\left\{0,1, \ldots, q^{j}-1\right\}
$$

Since by Cauchy schwarz inequality $\{\alpha(u(n)) \overline{\beta(u(n))}\}_{n \in \mathbb{N}_{0}} \in \ell^{1}(\mathcal{Z})$, therefore by invoking Lemma 3.1, (3.21) follows.

Theorem 3.2. Let $\widehat{V}$ be a subspace of $\ell^{2}(\mathcal{Z})$ that is invariant under the modulation operator $\mathcal{E}_{j}$ for all $j \in \mathbb{N}_{0}$. Then $\widehat{V}$ is dense in $\ell^{2}(\mathcal{Z})$ if and only if $\bigcap_{\widehat{V}}\{u(n) \in \mathcal{Z}: \alpha(u(n))=0\}$ is an empty set.

Proof. Assume that $\bigcap\{u(n) \in \mathcal{Z}: \alpha(u(n))=0\}$ is empty. It suffices to show that if $\alpha \in \widehat{V}$

$\beta \in \ell^{2}(\mathcal{Z})$ is orthogonal to $\widehat{V}$, then $\beta=0$. By using Lemma 3.2, for all $u(n) \in \mathcal{Z}$ and $\alpha \in \widehat{V}$,

$$
\alpha(u(n)) \overline{\beta(u(n))}=0 .
$$

Since

$$
\bigcap_{\alpha \in \widehat{V}}\{u(n) \in \mathcal{Z}: \alpha(u(n))=0\}
$$

is empty for any $u(n) \in \mathcal{Z}$, there exists $\alpha \in \widehat{V}$ such that $\alpha(u(n)) \neq 0$. Thus $\beta(u(n))=0$ for all $u(n) \in \mathcal{Z}$ and hence $\beta=0$.

Now suppose that

$$
\bigcap_{\alpha \in \hat{V}}\{u(n) \in \mathcal{Z}: \alpha(u(n))=0\}
$$

is non empty. Then there exists $u(k) \in \mathcal{Z}$ such that for all $\alpha \in \widehat{V}, \alpha(u(k))=0$. Let

$$
e_{k}=\{\delta(u(n)-u(k))\}_{n} \in \ell^{2}(\mathcal{Z})
$$

where $\delta$ is the discrete Dirac delta function. Hence for all $\alpha \in \widehat{V}$,

$$
\left\langle\alpha, e_{k}\right\rangle_{\ell^{2}(\mathcal{Z})}=0
$$

which shows that $\widehat{V}$ is not dense in $\ell^{2}(\mathcal{Z})$ and this is a contradiction. 
Corollary 3.4. For $j \in \mathbb{N}_{0}$, assume that $\widehat{V}_{j} \subseteq \widehat{V}_{j+1}$, where $\widehat{V}_{j}$ is defined as in (3.10). Then the union $\bigcup_{j=0}^{\infty} \widehat{V}_{j}$ is dense in $\ell^{2}(\mathcal{Z})$ if and only if $\bigcap_{j=0}^{\infty}\left\{u(n) \in \mathcal{Z}: \widehat{\varphi}_{j}(u(n))=0\right\}$ is an empty set.

Proof Let $\widehat{V}=\bigcup_{j=0}^{\infty} \widehat{V}_{j}$. Therefore by Proposition 3.7, we can say that $\widehat{V}$ is invariant under modulation operator $\mathcal{E}_{j}$ for all $j \in \mathbb{N}_{0}$. Further, we have

$$
\begin{aligned}
\bigcap_{\alpha \in \widehat{V}}\{u(n) \in \mathcal{Z}: \alpha(u(n))=0\} & =\left\{u(n) \in \mathcal{Z}: \alpha_{j, s}(u(n))=0, \forall j \in \mathbb{N}_{0}, s=0,1, \ldots, q^{j}-1\right\} \\
& =\left\{u(n) \in \mathcal{Z}: \hat{\varphi}_{j}(u(n))=0, \forall j \in \mathbb{N}_{0}\right\} .
\end{aligned}
$$

The process of constructing wavelet frames involves finding sequences $\widehat{\psi}_{j}^{\ell} \in \widehat{V}_{j+1}$ for $\ell=$ $1,2, \ldots, L$ such that the collection $\left\{\mathcal{E}_{j}^{s} \widehat{\psi}_{j}^{\ell}: 0 \leq s \leq q^{j}-1,1 \leq \ell \leq L\right\}$ is a frame for $\widehat{W}_{j}$, the orthogonal complement of $\widehat{V}_{j}$ in $\widehat{V}_{j+1}$, with frame bounds $C$ and $D$ independent of $j$. For $j \in N_{0}$ and $1 \leq \ell \leq L$, given the sequences $\widehat{\psi}_{j}^{\ell} \in \widehat{V}_{j+1}$, we define the orthogonal sequences $b_{j, s}^{\ell}$ for $0 \leq s \leq q^{j}-1$ by

$\beta_{j, s}^{\ell}(u(k)):=\left\{\begin{array}{l}\hat{\psi}_{j}^{\ell}(u(k)), \text { if } u(k)=\mathfrak{p}^{-j} u(p)+u(s), p \in \mathbb{N}_{0} \\ 0, \text { otherwise }\end{array}\right.$

Therefore, we have the decomposition $\widehat{\psi}_{j}^{\ell}=\sum_{s=0}^{q^{j}-1} \beta_{j, s}^{\ell}$ for $1 \leq \ell \leq L$.

Proposition 3.8. For every $j \in \mathbb{N}_{0}$, the sequence space $\widehat{W}_{j}$ is spanned by

$$
\left\{\mathcal{E}_{j}^{s} \hat{\psi}_{j}^{m}: 0 \leq s \leq q^{j}-1,1 \leq \ell \leq L\right\}
$$

if and only if it is spanned by $\left\{\beta_{j, s}^{\ell}: 0 \leq s \leq q^{j}-1,1 \leq \ell \leq L\right\}$.

Proof The proof is similar to that of Proposition 3.5.

Proposition 3.9 For every $j \in \mathbb{N}_{0}$, the following are equivalent:

(i) $\operatorname{span}\left\{\mathcal{E}_{j}^{s} \hat{\psi}_{j}^{m}: 0 \leq s \leq q^{j}-1,1 \leq \ell \leq L\right\} \subseteq \widehat{V}_{j+1}$.

(ii)There exists $g_{j+1}^{\ell} \in \mathcal{S}\left(\mathfrak{p}^{-(j+1)}\right)$ for $1 \leq \ell \leq L$ such that

$$
\hat{\psi}_{j}^{\ell}=\sum_{s=0}^{q^{j}-1} g_{j+1}^{\ell}(u(s)) \mathcal{E}_{j+1}^{s} \hat{\varphi}_{j+1}
$$

(iii) There exists $\widehat{g}_{j+1}^{\ell} \in \mathcal{S}\left(\mathfrak{p}^{-(j+1)}\right)$ for $1 \leq \ell \leq L$ such that for $0 \leq s \leq q^{j}-1$.

$$
\beta_{j, r}^{\ell}=\sum_{s=0}^{q-1} \hat{g}_{j+1}^{\ell}\left(\mathfrak{p}^{-j} u(s)+u(r)\right) \alpha_{j+1, \mathfrak{p}^{-j} u(s)+u(r)} .
$$

Proof The proof is similar to that of Proposition 3.6,

Now we shall restate Corollary 3.1 for the wavelet subspaces $\widehat{W}_{j}$ 's. 
Theorem 3.3. The necessary and sufficient condition for the collection $\left\{\mathcal{E}_{j}^{s} \widehat{\psi}_{j}^{m}: 0 \leq s \leq\right.$ $\left.q^{j}-1,1 \leq \ell \leq L\right\}$ to be a frame for $\widehat{W}_{j}$ with frame bounds $A$ and $B$ is that for each $0 \leq s \leq q^{j}-1$,

$$
\mathfrak{p}^{j} A \leq \lambda_{s}^{\ell} \leq \mathfrak{p}^{j} B
$$

for every nonzero eigen values of $\lambda_{s}^{\ell}, 1 \leq \ell \leq L$, of $\left[\left\langle\beta_{j, s}^{\ell}, \beta_{j, s}^{m}\right\rangle_{\ell^{2}(\mathcal{Z})}\right]_{\ell, m=1}^{L}$.

Proposition 3.10. Let $\widehat{V}_{j} \subseteq \widehat{V}_{j+1}$ and $\operatorname{span}\left\{\mathcal{E}_{j}^{s} \widehat{\psi}_{j}^{\ell}: 0 \leq s \leq q^{j}-1,1 \leq \ell \leq L\right\} \subseteq \widehat{V}_{j+1}$ for $j \in \mathbb{N}_{0}$. Then for $0 \leq s \leq q^{j}-1,1 \leq \ell, m \leq L$, the following holds:

$$
\begin{gathered}
\left\|\alpha_{j, r}\right\|_{\ell^{2}(\mathcal{Z})}^{2}=\sum_{s=0}^{q-1}\left|\hat{h}_{j+1}\left(\mathfrak{p}^{-j} u(s)+u(r)\right)\right|^{2}\left\|\alpha_{j+1, \mathfrak{p}^{-j} u(s)+u(r)}\right\|_{\ell^{2}(\mathcal{Z})}^{2}, \\
\left\langle\beta_{j, r}^{\ell}, \beta_{j, r}^{m}\right\rangle_{\ell^{2}(\mathcal{Z})}=\sum_{s=0}^{q-1} \hat{g}_{j+1}^{m}\left(\mathfrak{p}^{-j} u(s)+u(r)\right)\left\|\alpha_{j+1, \mathfrak{p}}-j u(s)+u(r)\right\|_{\ell^{2}(\mathcal{Z})}^{2} \overline{\hat{g}_{j+1}^{m}\left(\mathfrak{p}^{-j} u(s)+u(r)\right)}
\end{gathered}
$$

Proof. The proof follows from Propositions 3.6 and 3.9 .

Theorem 3.4. For $j \in \mathbb{N}_{0}$, assume that $\widehat{V}_{j} \subseteq \widehat{V}_{j+1}$ and that the union $\bigcup_{j=0}^{\infty} \widehat{V}_{j}$ is dense is $\ell^{2}(\mathcal{Z})$. Then the spaces $\widehat{W}_{j}^{\prime} s$ and $\widehat{V}_{0}$ are pairwise orthogonal and $\ell^{2}(\mathcal{Z})=\widehat{V}_{0} \oplus \bigoplus_{j=0}^{\infty} \widehat{W}_{j}$.

Proof For any $\alpha_{i} \in \widehat{W}_{i} \subseteq \widehat{V}_{i+1} \subseteq \widehat{V}_{j}$ and $\alpha_{j} \in \widehat{W}_{j}$, it is clear that for $i<j$ we have

$$
\left\langle\alpha_{i}, \alpha_{j}\right\rangle=0
$$

By definition, $\widehat{V}_{0}$ is orthogonal to the wavelet spaces $\widehat{W}_{j}^{\prime}$ s. Let $\mathcal{P}_{j}: \ell^{2}(\mathcal{Z}) \rightarrow \widehat{V}_{j}$ be the orthogonal projection operator from $\ell^{2}(\mathcal{Z})$ onto $\widehat{V}_{j}$. Then it clearly follows that

$$
\widehat{W}_{j}=\left\{\alpha-\mathcal{P}_{j} \alpha: \alpha \in \widehat{V}_{j+1}\right\} \text {. }
$$

Since $\alpha=\lim _{j \rightarrow \infty} \mathcal{P}_{j} \alpha$, therefore , for any $\alpha \in \ell^{2}(\mathcal{Z})$ we have

$$
\alpha=\mathcal{P}_{0} \alpha+\sum_{j=0}^{\infty}\left(\mathcal{P}_{j+1} \alpha-\mathcal{P}_{j} \alpha\right)
$$

Since $\mathcal{P}_{j+1}-\mathcal{P}_{j}$ is the orthogonal projector from $\ell^{2}(\mathcal{Z})$ onto $\widehat{W}_{j}$, therefore the desired result follows.

By invoking Proposition 3.5, for $j \in \mathbb{N}_{0}$, we may write $\widehat{V}_{j}$ as the orthogonal direct sum $\widehat{V}_{j}=\bigoplus_{r=0}^{q^{j}-1} \widehat{V}_{j, r}$, where $\widehat{V}_{j, r}=\operatorname{span}\left\{\alpha_{j, r}\right\}$ with dimension 0 or 1 for all $0 \leq r \leq q^{j}-1$. we may also write $\widehat{V}_{j+1}$ as the orthogonal direct $\operatorname{sum} \widehat{V}_{j+1}=\bigoplus_{r=0}^{q^{j}-1} \widetilde{V}_{j+1, r}$, where $\widetilde{V}_{j+1, r}=$ $\operatorname{span}\left\{\alpha_{j+1, \mathfrak{p}^{-j} u(s)+u(r)}: 0 \leq s \leq q-1\right\}$ with dimension between 0 and $q$ for all $0 \leq r \leq q^{j}-1$. Now we suppose that for $j \in \mathbb{N}_{0}$, the collection $\left\{\mathcal{E}_{j}^{r} \hat{\psi}_{j}^{\ell}: 0 \leq r \leq q^{j}-1,1 \leq \ell \leq L\right\}$ spans the wavelet subspace $\widehat{W}_{j}$, By using proposition 3.8, we shall write

$$
\widehat{W}_{j}=\bigoplus_{r=0}^{q^{j}-1} \widehat{W}_{j, r}
$$

where $\widehat{W}_{j, r}=\operatorname{span}\left\{\beta_{j, r}^{\ell}: 1 \leq \ell \leq L\right\}$ with dimension between 0 and $L$ for all $0 \leq r \leq q^{j}-1$.

On the basis of the orthogonal decomposition of the spaces $\widehat{V}_{j}$ and $\widehat{W}_{j}$ for $j \in \mathbb{N}_{0}$, we have a tool to characterize their orthogonal direct sum relationship with $\widehat{V}_{j+1}$. 
Proposition 3.11. For $j \in \mathbb{N}_{0}$, the sequence space $\widehat{V}_{j+1}$ can be expressed as the orthogonal direct sum $\widehat{V}_{j+1}=\widehat{V}_{j} \oplus \widehat{W}_{j}$ if and only if for every $0 \leq r \leq q^{j}-1$, the sequence space $\widetilde{V}_{j+1, r}$ can be expressed as the orthogonal direct sum $\widetilde{V}_{j+1, r}=\widehat{V}_{j, r} \oplus \widehat{W}_{j, r}$.

Proof. First, we assume that $\widehat{V}_{j+1}=\widehat{V}_{j} \oplus \widehat{W}_{j}$, therefore by using Propositions (3.6) and (3.10), we have

$$
\widehat{V}_{j, r} \oplus \widehat{W}_{j, r} \subset \widetilde{V}_{j+1, r} \text { for all } 0 \leq r \leq q^{j}-1
$$

Now let $\alpha \in \widetilde{V}_{j+1, r_{0}}$ for a given $r_{0} \in\left\{0,1,2, \ldots, q^{j}-1\right\}$. Then

$$
\begin{aligned}
\widetilde{V}_{j+1, r_{0}} & \subseteq \widehat{V}_{j+1} \\
& =\widehat{V}_{j} \oplus \widehat{W}_{j} \\
& =\left[\bigoplus_{r \in\left\{0,1, \ldots, q^{j}-1\right\}} \widehat{V}_{j, r}\right] \bigoplus\left[\bigoplus_{r \in\left\{0,1, \ldots, q^{j}-1\right\}} \widehat{W}_{j, r}\right] \\
& =\bigoplus_{r \in\left\{0,1, \ldots, q^{j}-1\right\} \backslash\left\{r_{0}\right\}}\left[\widehat{V}_{j, r} \bigoplus \widehat{W}_{j, r}\right] \bigoplus\left[\widehat{V}_{j, r_{0}} \oplus \widehat{W}_{j, r_{0}}\right] \\
& \subseteq\left[\bigoplus_{r \in\left\{0,1, \ldots, q^{j}-1\right\} \backslash\left\{r_{0}\right\}} \widetilde{V}_{j+1, r}\right] \bigoplus\left[\widehat{V}_{j, r_{0}} \oplus \widehat{W}_{j, r_{0}}\right] .
\end{aligned}
$$

Since $\widetilde{V}_{j+1, r}, r \in\left\{0,1, \ldots, q^{j}-1\right\} \backslash\left\{r_{0}\right\}$ and $\widehat{V}_{j, r_{0}} \oplus \widehat{W}_{j, r_{0}} \subseteq \widetilde{V}_{j+1, r_{0}}$ are pairwise orthogonal,therefore by (3.31) we can write $\alpha=\beta+\gamma$ where $\beta \in \bigoplus_{r \in\left\{0,1, \ldots, q^{j}-1\right\} \backslash\left\{r_{0}\right\}} \widetilde{V}_{j+1, r}$ and $\gamma \in \widehat{V}_{j, r_{0}} \oplus \widehat{W}_{j, r_{0}} \subseteq \widetilde{V}_{j+1, r_{0}}$ which implies that $\beta=\alpha-\gamma \in \widetilde{V}_{j+1, r_{0}}$. Then it is clear that $\beta=0$ and so $\alpha=\gamma$. Therefore for an arbitrary $r_{0} \in\left\{0,1, \ldots, q^{j}-1\right\}$ we have

$$
\widetilde{V}_{j+1, r_{0}} \subseteq \widehat{V}_{j, r_{0}} \oplus \widehat{W}_{j, r_{0}}
$$

By Combining (3.30) and (3.32) the result follows.

On the other hand, for all $r \in\left\{0,1, \ldots, q^{j}-1\right\}$, since $\widetilde{V}_{j+1, r}=\widehat{V}_{j, r} \oplus \widehat{W}_{j, r}$, we have the direct sum

$$
\bigoplus_{r \in\left\{0,1, \ldots, q^{j}-1\right\}} \widetilde{V}_{j+1, r}=\left[\bigoplus_{r \in\left\{0,1, \ldots, q^{j}-1\right\}} \widehat{V}_{j, r}\right] \bigoplus\left[\bigoplus_{r \in\left\{0,1, \ldots, q^{j}-1\right\}} \widehat{W}_{j, r}\right] .
$$

This implies,

$$
\widetilde{V}_{j+1, r}=\widehat{V}_{j, r} \oplus \widehat{W}_{j, r}
$$

Theorem 3.5. For $j \in \mathbb{N}_{0}$, assume that $\widehat{V}_{j} \subseteq \widehat{V}_{j+1}$, then the sequence spaces $\widehat{V}_{j, r}$ and $\widehat{W}_{j, r}$ are orthogonal if and only if for $0 \leq r \leq q^{j}-1,1 \leq \ell \leq L$,

$$
\sum_{s=0}^{q-1} \hat{h}_{j+1}\left(\mathfrak{p}^{-j} u(s)+u(r)\right)\left\|\alpha_{j+1, \mathfrak{p}^{-j} u(s)+u(r)}\right\|_{\ell^{2}(\mathcal{Z})}^{2} \overline{\hat{g}_{j+1}^{\ell}\left(\mathfrak{p}^{-j} u(s)+u(r)\right)}=0 .
$$

Furthermore, if (3.33) holds, the the sequence space $\widetilde{V}_{j+1, r}$ can be expressed as the orthogonal direct sum $\widetilde{V}_{j+1, r}=\widehat{V}_{j, r} \oplus \widehat{W}_{j, r}$ if and only if

$$
\operatorname{dim} \widehat{V}_{j+1, r}=\operatorname{dim} \widehat{V}_{j, r}+\operatorname{dim} \widehat{W}_{j, r} .
$$


Proof. From the definition of $\widehat{V}_{j, r}$ and $\widehat{W}_{j, r}$ and Propositions (3.6) and (3.9), the proof of (3.33) follows. Also the proof of (3.34) follows from the fact that $\widehat{V}_{j, r}+\widehat{W}_{j, r} \subseteq \widetilde{V}_{j+1, r}$ and $\operatorname{dim}\left(\widehat{V}_{j, r}+\widehat{W}_{j, r}\right)=\operatorname{dim} \widehat{V}_{j, r}+\operatorname{dim} \widehat{W}_{j, r}=\operatorname{dim} \widetilde{V}_{j+1, r}$

Theorem 3.6. Suppose that $\left\{\widehat{\varphi}_{j}, \widehat{V}_{j}: j \in \mathbb{N}_{0}\right\}$ is a frame multiresolution analysis of $\ell^{2}(\mathcal{Z})$. Then for $j \in \mathbb{N}_{0}$, there exists $\widehat{\psi}_{j}^{m} \in \widehat{V}_{j+1}$ for $1 \leq m \leq q$ such that

$$
\left\{\mathcal{E}_{j}^{s} \widehat{\psi}_{j}^{m}: 0 \leq s \leq q^{j}-1,1 \leq m \leq q\right\}
$$

forms a tight frame for $\widehat{W}_{j}$ with frame bound 1 . Therefore, the collection

$$
\left\{\mathcal{E}_{j}^{s} \widehat{\psi}_{j}^{m}: 0 \leq s \leq q^{j}-1,1 \leq m \leq q, j \in \mathbb{N}_{0}\right\} \cup\left\{\widehat{\varphi}_{0}\right\}
$$

is a frame for $\ell^{2}(\mathcal{Z})$.

Proof. Fix $j \in \mathbb{N}_{0}$. In our procedure to construct wavelet frames, we are interested in the cases when $\operatorname{dim} \widehat{W}_{j}>0$ and we shall proceed by determining $\widehat{g}_{j+1}\left(\mathfrak{p}^{-j} u(\ell)+u(s)\right)$ for $1 \leq m \leq L, 0 \leq \ell \leq q-1$ and $0 \leq s \leq q^{j}-1$, satisfying (3.33), (3.34) and $\left\langle\beta_{j, s}^{m}, \beta_{j, s}^{n}\right\rangle=0$ for $1 \leq m, n \leq q$.

Suppose that $\operatorname{dim} \widetilde{V}_{j+1, s}=p$ for some $1 \leq p \leq q$ and $\operatorname{dim} \widehat{V}_{j, s}=\lambda$ for some $\lambda=0$ or 1. Therefore, we need $\operatorname{dim} \widehat{W}_{j, s}=p-\lambda$. If $p-\lambda=0$, then we proceed to (3.37) and ignore (3.38). Let $\left\{\ell_{0}, \ell_{1}, \ldots, \ell_{q-1}\right\}=\Delta_{q}$ such that

$$
\begin{gathered}
\left\|\alpha_{j+1, \mathfrak{p}^{-j} \ell_{k}+s}\right\|^{2}>0 \text { for } k \in\{0,1, \ldots, p-1\}, \\
\left\|\alpha_{j+1, \mathfrak{p}^{-j} \ell_{k}+s}\right\|^{2}>0 \text { for } k \in \mid \text { delta }_{q} \backslash\{0,1, \ldots, p-1\} .
\end{gathered}
$$

By corollary 3.2 , for $k \in\{0,1,2, \ldots p-1\}$, we have

$$
\mathfrak{p}^{j+1} A \leq\left\|\alpha_{j+1, \mathfrak{p}^{-j} \ell_{k}+s}\right\|^{2} \leq \mathfrak{p}^{j+1} B .
$$

Consider the $p \times 1$ matrix

$$
V=\frac{1}{\sqrt{q}}\left[\left\|\alpha_{j+1, \mathfrak{p}^{-j} \ell_{k}+s}\right\|^{2} \widehat{h}\left(\mathfrak{p}^{-j} \ell_{k}+u(s)\right)\right]_{k=0}^{p-1} .
$$

Then there exists an unitary $p \times p$ matrix $U$ such that

$$
U V=\left[\|V\|_{\mathbb{C}^{p}} O_{(p-1) \times 1}\right]^{T} .
$$

If $V \neq 0$, then by Proposition 3.10, we have $\left\|\alpha_{j, s}\right\|^{2}=0$ and hence $\lambda=1$. In this case we set

$$
U=\left[\begin{array}{ll}
U_{1} & U_{2}
\end{array}\right]^{T}
$$

where $U_{1}$ is a $1 \times p$ matrix and $U_{2}$ is a $(p-1) \times p$ matrix.

If $V=0$, then by Proposition 3.10, we have $\left\|\alpha_{j, s}\right\|^{2}=0$ and hence $\lambda=0$. In this case we set $U_{2}=U$.

Now we shall define $\widehat{g}_{j+1}^{m}\left(\mathfrak{p}^{-j} \ell_{k}+u(s)\right)$ for $1 \leq m \leq L, 0 \leq k \leq q-1$. For $1 \leq m \leq p-\lambda$ and $0 \leq k \leq p-1$, we define $\widehat{g}_{j+1}^{m}\left(\mathfrak{p}^{-j} \ell_{k}+u(s)\right)$ such that

$$
U_{2}=\sqrt{q^{j}}\left[\overline{\widehat{g}_{j+1}^{m}\left(\mathfrak{p}^{-j} \ell_{k}+u(s)\right)}\left\|\alpha_{j+1, \mathfrak{p}^{-j} \ell_{k}+s}\right\|^{2}\right]_{m=1, k=0}^{p-\lambda, p-1}
$$


For $p-\lambda+1 \leq m \leq L$ and $0 \leq k \leq p-1$, we define

$$
\widehat{g}_{j+1}^{m}\left(\mathfrak{p}^{-j} \ell_{k}+u(s)\right)=0 .
$$

For $1 \leq m \leq L$, and $k \in \Delta_{p} \backslash\{0,1, \ldots p-1\}$, we define $\widehat{g}_{j+1}^{m}\left(\mathfrak{p}^{-j} \ell_{k}+u(s)\right)$ arbitrarily.

Since $U_{2} V=0$, we have

$$
\sum_{k=0}^{p-1} \widehat{h}_{j+1}\left(\mathfrak{p}^{-j} \ell_{k}+u(s)\right)\left\|\alpha_{j+1, \mathfrak{p}^{-j} \ell_{k}+u(r)}\right\|^{2} \overline{\widehat{g}_{j+1}^{m}\left(\mathfrak{p}^{-j} \ell_{k}+u(s)\right)}=0,
$$

for $1 \leq m \leq p-\lambda$. By (3.37), we also have

$$
\sum_{k=0}^{p-1} \widehat{h}_{j+1}\left(\mathfrak{p}^{-j} \ell_{k}+u(s)\right)\left\|\alpha_{j+1, \mathfrak{p}^{-j} \ell_{k}+u(s)}\right\|^{2} \overline{\widehat{g}_{j+1}^{m}\left(\mathfrak{p}^{-j} \ell_{k}+u(s)\right)}=0,
$$

for $1 \leq m \leq L$. Further, by (3.35) we obtain

$$
\sum_{k \in \Delta_{q}} \widehat{h}_{j+1}\left(\mathfrak{p}^{-j} \ell_{k}+u(r)\right)\left\|\alpha_{j+1, \mathfrak{p}^{-j} \ell_{k}+u(r)}\right\|^{2} \overline{\widehat{g}_{j+1}\left(\mathfrak{p}^{-j} \ell_{k}+u(s)\right)}=0
$$

where $\widehat{g}_{j+1}=\left[\widehat{g}_{j+1}^{1}, \widehat{g}_{j+1}^{2}, \ldots, \widehat{g}_{j+1}^{L}\right]$.

Since $U$ is an unitary matrix, the rows of $U_{2}$ are orthonormal. Therefore, $U_{2} U_{2}^{*}=I$. Combining with (3.35), this gives

$$
\sum_{k \in \Delta_{q}} \widehat{g}_{j+1}^{m}\left(\mathfrak{p}^{-j} \ell_{k}+u(s)\right)\left\|\alpha_{j+1, \mathfrak{p}^{-j} \ell_{k}+u(r)}\right\|^{2} \overline{\widehat{g}_{j+1}^{n}\left(\mathfrak{p}^{-j} \ell_{k}+u(s)\right)}=\frac{\delta_{m n}}{q^{j}}
$$

for $1 \leq m, n \leq p-\lambda$. By (3.37), we have

$$
\sum_{k \in \Delta_{q}} \widehat{g}_{j+1}^{m}\left(\mathfrak{p}^{-j} \ell_{k}+u(s)\right)\left\|\alpha_{j+1, \mathfrak{p}^{-j} \ell_{k}+u(s)}\right\|^{2} \overline{\widehat{g}_{j+1}^{n}\left(\mathfrak{p}^{-j} \ell_{k}+u(s)\right)}=0
$$

for $p-\lambda+1 \leq m, m \leq L$. By Proposition 3.10 and Eqns (3.41) and (3.42), we conclude that

$$
\begin{gathered}
\left\|\beta_{j, s}^{m}\right\|^{2}=\frac{1}{q^{j}} \text { for } 1 \leq m \leq p-\lambda, \\
\left\|\beta_{j, s}^{m}\right\|^{2}=0 \text { for } p-\lambda+1 \leq m \leq L,
\end{gathered}
$$

Therefore, $\operatorname{dim} \widehat{W}_{j, s}=p-\lambda$ and by invoking Theorem 3.3 and Proposition 3.10,

$$
\left\{\mathcal{E}_{j}^{s} \widehat{\psi}_{j}^{m}: 0 \leq s \leq q^{j}-1,1 \leq m \leq L\right\}
$$

forms a tight frame for $\widehat{W}_{j}$ with frame bound 1 . For the case when $p=0$, there is no restriction to the values of $\widehat{g}_{j+1}^{m}\left(\mathfrak{p}^{-j} \ell_{k}+u(s)\right)$ for $1 \leq m \leq L$ and $k \in \Delta_{q}$ except that they are finite. By invoking Theorem 3.4, the collection

$$
\left\{\mathcal{E}_{j}^{s} \widehat{\psi}_{j}^{m}: 0 \leq s \leq q^{j}-1,1 \leq m \leq q, j \in \mathbb{N}_{0}\right\} \cup\left\{\widehat{\varphi}_{0}\right\}
$$

is a frame for $\ell^{2}(\mathcal{Z})$.

Corollary 3.5. Suppose that $\left\{\widehat{\varphi}_{j}, \widehat{V}_{j}: j \in \mathbb{N}_{0}\right\}$ is a frame multiresolution analysis of $\ell^{2}(\mathcal{Z})$. Then for $j \in \mathbb{N}_{0}$, there exists $\widehat{\psi}_{j}^{m} \in \widehat{V}_{j+1}$ for $1 \leq m \leq q$ such that

$$
\left\{\mathcal{E}_{j}^{s} \widehat{\psi}_{j}^{m}: 0 \leq s \leq q^{j}-1,1 \leq m \leq \kappa_{j}\right\}
$$


forms a tight frame for $\widehat{W}_{j}$, where

$$
\kappa_{j}=\max _{0 \leq s \leq q^{j}-1}\left\{\operatorname{dim} \widetilde{V}_{j+1, s}-\operatorname{dim} \widehat{V}_{j, s}\right\} .
$$

Therefore, the collection

$$
\left\{\mathcal{E}_{j}^{s} \widehat{\psi}_{j}^{m}: 0 \leq s \leq q^{j}-1,1 \leq m \leq \kappa_{j}, j \in \mathbb{N}_{0}\right\} \cup\left\{\widehat{\varphi}_{0}\right\}
$$

is a frame for $\ell^{2}(\mathcal{Z})$.

Proof. Clearly $\kappa_{j}$ is bounded above by $q$ as $\operatorname{dim} \widetilde{V}_{j+1, s} \leq q$. By our construction, for $\kappa_{j}+1 \leq$ $m \leq q$ and $0 \leq s \leq q^{j}-1$. Since $m \geq \kappa_{j}+1 \geq \operatorname{dim} \widetilde{V}_{j+1, s}-\operatorname{dim} \widehat{V}_{j, s}$, it follows from Proposition 3.10 and Eqn (3.42) that $\left\|\beta_{j, s}^{m}\right\|^{2}=0$. Thus for $\kappa_{j}+1 \leq m \leq q, \widehat{\psi}_{j}^{m}=0$. By our construction, the proof follows from Theorem 3.6 by setting $L=\kappa_{j}$.

By invoking corollary 3.5, it is quite possible to use less number of sequences $\widehat{\varphi}_{j}^{m}$ to obtain a tight frame for $\widehat{W}_{j}$. In order to do so, we need the set

$$
\bigwedge=\left\{s \in\left\{0,1,2, \ldots, q^{j}-1\right\}: \alpha_{j, s}=0, \alpha_{j+1, \mathfrak{p}^{-j} \ell+s} \neq 0 \text { for all } 0 \leq m \leq q-1\right\}
$$

to be an empty set for a given $j \in \mathbb{N}_{0}$.

Corollary 3.5. Suppose that $\left\{\widehat{\varphi}_{j}, \widehat{V}_{j}: j \in \mathbb{N}_{0}\right\}$ is a frame multiresolution analysis of $\ell^{2}(\mathcal{Z})$. Then for $j \in \mathbb{N}_{0}$, there exists $\widehat{\psi}_{j}^{m} \in \widehat{V}_{j+1}$ for $1 \leq m \leq q$ such that

$$
\left\{\mathcal{E}_{j}^{s} \widehat{\psi}_{j}^{m}: 0 \leq s \leq q^{j}-1,1 \leq m \leq q-1\right\}
$$

forms a tight frame for $\widehat{W}_{j}$, if and only if the set $\Lambda$ defined in (3.45) is empty. If the latter holds for all $j \in \mathbb{N}_{0}$, then the collection

$$
\left\{\mathcal{E}_{j}^{s} \widehat{\psi}_{j}^{m}: 0 \leq s \leq q^{j}-1,1 \leq m \leq q-1, j \in \mathbb{N}_{0}\right\} \cup\left\{\widehat{\varphi}_{0}\right\}
$$

is a frame for $\ell^{2}(\mathcal{Z})$.

Proof If $\bigwedge$ is empty, then necessarily for all $s \in\left\{0,1,2, \ldots q^{j}-1\right\}, \operatorname{dim} \widetilde{V}_{j+1, s}-\operatorname{dim} \widehat{V}_{j, s} \leq q-1$ as there does not exist $s \in\left\{0,1,2, \ldots q^{j}-1\right\}$ such that $\operatorname{dim} \widetilde{V}_{j+1, s}=q$ and $\operatorname{dim} \widehat{V}_{j, s}=0$. Therefore, it folls that $\kappa_{j} \leq q-1$, where $\kappa_{j}$ is as defined in corollary 3.5. This proves the necessary part of the theorem.

If $\Lambda$ is not empty, then by definition of $\widetilde{V}_{j+1, s}$ and $\widehat{V}_{j, s}$, there exists $s \in\left\{0,1, \ldots, q^{j}-1\right\}$ such that $\operatorname{dim} \widetilde{V}_{j+1, s}=q$ and $\operatorname{dim} \widehat{V}_{j, s}=0$. Therefore, this requires $\operatorname{dim} \widehat{W}_{j, s}=q$ and thus $q-1 \widehat{\psi}_{j}^{m}$ 's are not sufficient.

\section{REFERENCES}

[1] O. Ahmad, M.Y. Bhat, N.A. Sheikh, Construction of Parseval Framelets Associated with GMRA on Local Fields of Positive Characteristic, Numer. Funct. Anal. Optim. 42 (2021) 344-370. https://doi.org/10. 1080/01630563.2021.1878370.

[2] O. Ahmad, N. Ahmad, Construction of Nonuniform Wavelet Frames on Non-Archimedean Fields, Math. Phys. Anal. Geom. 23 (2020) 47. https://doi.org/10.1007/s11040-020-09371-1.

[3] O. Ahmad, N.A. Sheikh, K.S. Nisar, F.A. Shah, Biorthogonal wavelets on the spectrum, Math Meth Appl Sci. 44 (2021) 4479-4490. https://doi.org/10.1002/mma.7046. 
[4] O. Ahmad, Construction of nonuniform periodic wavelet frames on non-Archimedean fields, Ann. Univ. Mariae Curie-Skłodowska, Sect. A. 74 (2020) 1-17. https://doi.org/10.17951/a.2020.74.2.1-17.

[5] O. Ahmad, N. A Sheikh, Explicit Construction of Tight Nonuniform Framelet Packets on Local Fields, Oper. Matrices. 15 (2021) 131-149. https://doi.org/10.7153/oam-2021-15-10.

[6] O. Ahmad, N.A. Sheikh, M. A. Ali, Nonuniform nonhomogeneous dual wavelet frames in Sobolev spaces in $L^{2}(\mathbb{K})$, Afr. Math. 31 (2020) 1145-1156. https://doi.org/10.1007/s13370-020-00786-1.

[7] O. Ahmad and N. A. Sheikh, On Characterization of nonuniform tight wavelet frames on local fields, Anal. Theory Appl. 34 (2018) 135-146. https://doi.org/10.4208/ata.2018.v34.n2.4.

[8] O. Ahmad, F.A. Shah, N.A. Sheikh, Gabor frames on non-Archimedean fields, Int. J. Geom. Methods Mod. Phys. 15 (2018) 1850079. https://doi.org/10.1142/S0219887818500792.

[9] S. Albeverio, S. Evdokimov, and M. Skopina, $p$-adic nonorthogonal wavelet bases, Proc. Steklov Inst. Math. 265 (2009) 1-12.

[10] S. Albeverio, S. Evdokimov, M. Skopina, p-Adic Multiresolution Analysis and Wavelet Frames, J. Fourier Anal. Appl. 16 (2010) 693-714. https://doi.org/10.1007/s00041-009-9118-5.

[11] S. Albeverio, A. Khrennikov, and V. Shelkovich, Theory of p-adic Distributions: Linear and Nonlinear Models, Cambridge University Press, 2010.

[12] J. J. Benedetto and R. L. Benedetto, A wavelet theory for local fields and related groups, J. Geom. Anal. 14 (2004) 423-456.

[13] O. Christensen, S.S. Goh, The unitary extension principle on locally compact abelian groups, Appl. Comput. Harm. Anal. 47 (2019) 1-29. https://doi.org/10.1016/j.acha.2017.07.004.

[14] R. J. Duffin and A. C. Shaeffer, A class of nonharmonic Fourier series, Trans. Amer. Math. Soc. 72 (1952) 341-366.

[15] S. Evdokimov and M. Skopina, 2-adic wavelet bases, Proc. Steklov Inst. Math. 266 (2009), S143-S154

[16] Y. Farkov, Orthogonal wavelets on locally compact abelian groups, Funct. Anal. Appl. 31 (1997), 294-296.

[17] Y. Farkov, Multiresolution Analysis and Wavelets on Vilenkin Groups, Facta Univ. (NIS), Ser.: Elec. Energ. 21 (2008) 309-325.

[18] H. Jiang, D. Li, N. Jin, Multiresolution analysis on local fields, J. Math. Anal. Appl. 294 (2004) 523-532.

[19] A. Khrennikov and V. Shelkovich, Non-Haar $p$-adic wavelets and their application to pseudo-differential operators and equations, Appl. Comput. Harmon. Anal. 28 (2010) 1-23. https://doi.org/10.1016/j. acha.2009.05.007.

[20] A. Khrennikov, V. Shelkovich, and M. Skopina, p-adic refinable functions and MRA-based wavelets, J. Approx. Theory. 161 (2009) 226-238. https://doi.org/10.1016/j.jat.2008.08.008.

[21] S. Kozyrev and A. Khrennikov, p-adic integral operators in wavelet bases, Doklady Math. 83 (2011) 209-212.

[22] S. Kozyrev, A. Khrennikov, and V. Shelkovich, $p$-Adic wavelets and their applications, Proc. Steklov Inst. Math. 285 (2014) 157-196.

[23] W. C. Lang, Orthogonal wavelets on the Cantor dyadic group, SIAM J. Math. Anal. 27 (1996) 305-312.

[24] W. C. Lang, Wavelet analysis on the Cantor dyadic group, Houston J. Math. 24 (1998) 533-544.

[25] W.C. Lang, Fractal multiwavelets related to the cantor dyadic group, Int. J. Math. Math. Sci. 21 (1998) 307-314. https://doi.org/10.1155/S0161171298000428.

[26] D. Li, H. Jiang, The necessary condition and sufficient conditions for wavelet frame on local fields, J. Math. Anal. Appl. 345 (2008) 500-510. https://doi.org/10.1016/j.jmaa.2008.04.031.

[27] S. G. Mallat, Multiresolution approximations and wavelet orthonormal bases of $L^{2}(\mathbb{R})$, Trans. Amer. Math. Soc. 315 (1989) 69-87.

[28] A. Ron and Z. Shen, Affine systems in $L^{2}\left(\mathbb{R}^{d}\right)$ : the analysis of the analysis operator, J. Funct. Anal. 148 (1997) $408-447$.

[29] F.A. Shah, O. Ahmad, Wave packet systems on local fields, J. Geom. Phys. 120 (2017) 5-18. https: //doi.org/10.1016/j.geomphys.2017.05.015.

[30] F. A. Shah, O. Ahmad and A. Rahimi, Frames Associated with Shift Invariant Spaces on Local Fields, Filomat. 32 (2018) 3097-3110. https://doi.org/10.2298/FIL1809097S.

[31] F. A. Shah, O. Ahmad and N. A. Sheikh, Orthogonal Gabor systems on local fields. Filomat 31 (2017) 5193-5201. https://doi.org/10.2298/FIL1716193S. 
[32] F. A. Shah, O. Ahmad and N. A. Sheikh, Some new inequalities for wavelet frames on local fields. Anal. Theory Appl. 33 (2017) 134-148. https://doi.org/10.4208/ata.2017.v33.n2.4.

[33] M. H. Taibleson, Fourier Analysis on Local Fields, Princeton University Press, Princeton, NJ, 1975. 